\title{
FIVE-YEAR WILKINSON MICROWAVE ANISOTROPY PROBE* OBSERVATIONS: DATA PROCESSING, SKY MAPS, AND BASIC RESULTS
}

\author{
G. Hinshaw ${ }^{1}$, J. L. Weiland ${ }^{2}$, R. S. Hill ${ }^{2}$, N. Odegard ${ }^{2}$, D. Larson ${ }^{3}$, C. L. Bennett ${ }^{3}$, J. Dunkley ${ }^{4,5,6}$, B. Gold ${ }^{3}$, \\ M. R. Greason ${ }^{2}$, N. JarosiK ${ }^{4}$, E. Komatsu ${ }^{7}$, M. R. Nolta ${ }^{8}$, L. Page ${ }^{4}$, D. N. SPergel ${ }^{5,9}$, E. Wollack ${ }^{1}$, M. HalPern ${ }^{10}$, \\ A. Kogut ${ }^{1}$, M. Limon ${ }^{11}$, S. S. Meyer ${ }^{12}$, G. S. TuCKer ${ }^{13}$, And E. L. Wright ${ }^{14}$ \\ ${ }^{1}$ Code 665, NASA/Goddard Space Flight Center, Greenbelt, MD 20771, USA; Gary.F.Hinshaw@nasa.gov \\ 2 Adnet Systems, Inc., 7515 Mission Dr., Suite A100, Lanham, MD 20706, USA \\ ${ }^{3}$ Department of Physics \& Astronomy, The Johns Hopkins University, 3400 N. Charles St., Baltimore, MD 21218-2686, USA \\ ${ }^{4}$ Department of Physics, Jadwin Hall, Princeton University, Princeton, NJ 08544-0708, USA \\ ${ }^{5}$ Department of Astrophysical Sciences, Peyton Hall, Princeton University, Princeton, NJ 08544-1001, USA \\ ${ }^{6}$ Astrophysics, University of Oxford, Keble Road, Oxford, OX1 3RH, UK \\ ${ }^{7}$ Department of Astronomy, University of Texas, Austin, 2511 Speedway, RLM 15.306, Austin, TX 78712, USA \\ ${ }^{8}$ Canadian Institute for Theoretical Astrophysics, 60 St. George St, University of Toronto, Toronto, ON M5S 3H8, Canada \\ ${ }^{9}$ Princeton Center for Theoretical Physics, Princeton University, Princeton, NJ 08544, USA \\ ${ }^{10}$ Department of Physics and Astronomy, University of British Columbia, Vancouver BC V6T 1Z1, Canada \\ ${ }^{11}$ Columbia Astrophysics Laboratory, 550 W. 120th St., Mail Code 5247, New York, NY 10027-6902, USA \\ ${ }^{12}$ Departments of Astrophysics and Physics, KICP and EFI, University of Chicago, Chicago, IL 60637, USA \\ ${ }^{13}$ Department of Physics, Brown University, 182 Hope St., Providence, RI 02912-1843, USA \\ ${ }^{14}$ UCLA Physics \& Astronomy, P.O. Box 951547, Los Angeles, CA 90095-1547, USA \\ Received 2008 March 5; accepted 2008 September 8; published 2009 February 11
}

\begin{abstract}
We present new full-sky temperature and polarization maps in five frequency bands from 23 to $94 \mathrm{GHz}$, based on data from the first five years of the Wilkinson Microwave Anisotropy Probe (WMAP) sky survey. The new maps are consistent with previous maps and are more sensitive. The five-year maps incorporate several improvements in data processing made possible by the additional years of data and by a more complete analysis of the instrument calibration and in-flight beam response. We present several new tests for systematic errors in the polarization data and conclude that W-band polarization data is not yet suitable for cosmological studies, but we suggest directions for further study. We do find that Ka-band data is suitable for use; in conjunction with the additional years of data, the addition of Ka band to the previously used Q- and V-band channels significantly reduces the uncertainty in the optical depth parameter, $\tau$. Further scientific results from the five-year data analysis are presented in six companion papers and are summarized in Section 7 of this paper. With the five-year WMAP data, we detect no convincing deviations from the minimal six-parameter $\Lambda C D M$ model: a flat universe dominated by a cosmological constant, with adiabatic and nearly scale-invariant Gaussian fluctuations. Using WMAP data combined with measurements of Type Ia supernovae and Baryon Acoustic Oscillations in the galaxy distribution, we find (68\% CL uncertainties): $\Omega_{b} h^{2}=0.02267_{-0.00059}^{+0.00058}, \Omega_{c} h^{2}=0.1131 \pm 0.0034, \Omega_{\Lambda}=0.726 \pm 0.015$, $n_{s}=0.960 \pm 0.013, \tau=0.084 \pm 0.016$, and $\Delta_{\mathcal{R}}^{2}=(2.445 \pm 0.096) \times 10^{-9}$ at $k=0.002 \mathrm{Mpc}^{-1}$. From these we derive $\sigma_{8}=0.812 \pm 0.026, H_{0}=70.5 \pm 1.3 \mathrm{~km} \mathrm{~s}^{-1} \mathrm{Mpc}^{-1}, \Omega_{b}=0.0456 \pm 0.0015, \Omega_{c}=0.228 \pm 0.013$, $\Omega_{m} h^{2}=0.1358_{-0.0036}^{+0.0037}, z_{\text {reion }}=10.9 \pm 1.4$, and $t_{0}=13.72 \pm 0.12 \mathrm{Gyr}$. The new limit on the tensor-to-scalar ratio is $r<0.22(95 \% \mathrm{CL})$, while the evidence for a running spectral index is insignificant, $d n_{s} / d \ln k=-0.028 \pm 0.020$ (68\% CL). We obtain tight, simultaneous limits on the (constant) dark energy equation of state and the spatial curvature of the universe: $-0.14<1+w<0.12$ (95\% CL) and $-0.0179<\Omega_{k}<0.0081$ (95\% CL). The number of relativistic degrees of freedom, expressed in units of the effective number of neutrino species, is found to be $N_{\text {eff }}=4.4 \pm 1.5(68 \% \mathrm{CL})$, consistent with the standard value of 3.04. Models with $N_{\text {eff }}=0$ are disfavored at $>99.5 \%$ confidence. Finally, new limits on physically motivated primordial non-Gaussianity parameters are $-9<f_{\mathrm{NL}}^{\text {local }}<111(95 \% \mathrm{CL})$ and $-151<f_{\mathrm{NL}}^{\text {equil }}<253(95 \% \mathrm{CL})$ for the local and equilateral models, respectively.
\end{abstract} Key words: cosmic microwave background - cosmology: observations - early universe - dark matter - space vehicles - space vehicles: instruments - instrumentation: detectors - telescopes

\section{INTRODUCTION}

The Wilkinson Microwave Anisotropy Probe (WMAP) is a Medium-Class Explorer (MIDEX) satellite aimed at elucidating cosmology through full-sky observations of the cosmic microwave background (CMB). The WMAP full-sky maps of the temperature and polarization anisotropy in five frequency bands provide our most accurate view to date of conditions in the

* WMAP is the result of a partnership between Princeton University and NASA's Goddard Space Flight Center. Scientific guidance is provided by the WMAP Science Team. early universe. The multifrequency data facilitate the separation of the CMB signal from foreground emission arising both from our Galaxy and from extragalactic sources. The CMB angular power spectrum derived from these maps exhibits a highly coherent acoustic peak structure which makes it possible to extract a wealth of information about the composition and history of the universe, as well as the processes that seeded the fluctuations.

WMAP data (Bennett et al. 2003; Spergel et al. 2003, 2007; Hinshaw et al. 2007), along with a host of pioneering CMB experiments (Miller et al. 1999; Lee et al. 2001; Netterfield 
et al. 2002; Halverson et al. 2002; Pearson et al. 2003; Scott et al. 2003; Benoît et al. 2003), and other cosmological measurements (Percival et al. 2001; Tegmark et al. 2004, 2006; Cole et al. 2005; Eisenstein et al. 2005; Percival et al. 2007; Astier et al. 2006; Riess et al. 2007; Wood-Vasey et al. 2007) have established $\Lambda \mathrm{CDM}$ as the standard model of cosmology: a flat universe dominated by dark energy, supplemented by dark matter and atoms with density fluctuations seeded by a Gaussian, adiabatic, nearly scale invariant process. The basic properties of this universe are determined by five numbers: the density of matter, the density of atoms, the age of the universe (or equivalently, the Hubble constant today), the amplitude of the initial fluctuations, and their scale dependence.

By accurately measuring the first few peaks in the angular power spectrum and the large-scale polarization anisotropy, $W M A P$ data have enabled the following inferences:

1. A precise $(3 \%)$ determination of the density of atoms in the universe. The agreement between the atomic density derived from WMAP and the density inferred from the deuterium abundance is an important test of the standard big bang model.

2. A precise $(3 \%)$ determination of the dark matter density (with five years of data and a better determination of our beam response, this measurement has improved significantly). Previous CMB measurements have shown that the dark matter must be nonbaryonic and interact only weakly with atoms and radiation. The WMAP measurement of the density puts important constraints on supersymmetric dark matter models and on the properties of other dark matter candidates.

3. A definitive determination of the acoustic scale at redshift $z=1090$. Similarly, the recent measurement of baryon acoustic oscillations (BAOs) in the galaxy power spectrum (Eisenstein et al. 2005) has determined the acoustic scale at redshift $z \sim 0.35$. When combined, these standard rulers accurately measure the geometry of the universe and the properties of the dark energy. These data require a nearly flat universe dominated by dark energy consistent with a cosmological constant.

4. A precise determination of the Hubble Constant, in conjunction with BAO observations. Even when allowing curvature $\left(\Omega_{0} \neq 1\right)$ and a free dark energy equation of state $(w \neq-1)$, the acoustic data determine the Hubble constant to within $3 \%$. The measured value is in excellent agreement with independent results from the Hubble Key Project (Freedman et al. 2001), providing yet another important consistency test for the standard model.

5. Significant constraint of the basic properties of the primordial fluctuations. The anticorrelation seen in the temperature/polarization (TE) correlation spectrum on $4^{\circ}$ scales implies that the fluctuations are primarily adiabatic and rule out defect models and isocurvature models as the primary source of fluctuations (Peiris et al. 2003).

Further, the WMAP measurement of the primordial power spectrum of matter fluctuations constrains the physics of inflation, our best model for the origin of these fluctuations. Specifically, the five-year data provide the best measurement to date of the scalar spectrum's amplitude and slope, and place the most stringent limits to date on the amplitude of tensor fluctuations. However, it should be noted that these constraints assume a smooth function of scale, $k$. Certain models with localized structure in $P(k)$, and hence additional parameters, are not ruled out, neither are they required by the data (see e.g. Shafieloo \& Souradeep 2008; Hunt \& Sarkar 2007).

The statistical properties of the CMB fluctuations measured by WMAP are close to Gaussian; however, there are several hints of possible deviations from Gaussianity, e.g. Eriksen et al. (2007a); Copi et al. (2007); Land \& Magueijo (2007); Yadav $\&$ Wandelt (2008). Significant deviations would be a very important signature of new physics in the early universe.

Large-angular-scale polarization measurements currently provide our best window into the universe at $z \sim 10$. The WMAP data imply that the universe was reionized long before the epoch of the oldest known quasars. By accurately constraining the optical depth of the universe, WMAP not only constrains the age of the first stars but also determines the amplitude of primordial fluctuations to better than $3 \%$. This result is important for constraining the growth rate of structure.

This paper summarizes results compiled from five years of WMAP data that are fully presented in a suite of seven papers (including this one). The new results improve upon previous results in many ways: additional data reduce the random noise, which is especially important for studying the temperature signal on small angular scales and the polarization signal on large angular scales; five independent years of data enable comparisons and null tests that were not previously possible; the instrument calibration and beam response have been much better characterized, due in part to improved analyses and to additional years of data; and, other cosmological data have become available.

In addition to summarizing the other papers, this paper reports on changes in the WMAP data processing pipeline, presents the five-year temperature and polarization maps, and gives new results on instrument calibration and on potential systematic errors in the polarization data. Hill et al. (2009) discuss the program to derive an improved physical optics model of the WMAP telescope, and use the results to better determine the WMAP beam response. Gold et al. (2009) present a new analysis of diffuse foreground emission in the WMAP data and update previous analyses using five-year data. Wright et al. (2009) analyze extragalactic point sources and provide an updated source catalog, with new results on source variability. Nolta et al. (2009) derive the angular power spectra from the maps, including the TT, TE, TB, EE, EB, and BB spectra. Dunkley et al. (2009) produce an updated likelihood function and present cosmological parameter results based on fiveyear WMAP data. They also develop an independent analysis of polarized foregrounds and use those results to test the reliability of the optical depth inference to foreground removal errors. Komatsu et al. (2009) infer cosmological parameters by combining five-year WMAP data with a host of other cosmological data and discuss the implications of the results. Concurrent with the submission of these papers, all five-year WMAP data are made available to the research community via NASA's Legacy Archive for Microwave Background Data Analysis (LAMBDA). The data products are described in detail in the WMAP Explanatory Supplement (Limon et al. 2008), which is also available on LAMBDA.

The WMAP instrument is composed of ten differencing assemblies (DAs) spanning five frequencies from 23 to $94 \mathrm{GHz}$ (Bennett et al. 2003): one DA each at $23 \mathrm{GHz}$ (K1) and $33 \mathrm{GHz}$ (Ka1), two each at $41 \mathrm{GHz}(\mathrm{Q} 1, \mathrm{Q} 2)$ and 61 $\mathrm{GHz}(\mathrm{V} 1, \mathrm{~V} 2)$, and four at $94 \mathrm{GHz}(\mathrm{W} 1-\mathrm{W} 4)$. Each DA is formed from two differential radiometers which are sensitive to orthogonal linear polarization modes; the radiometers are 
Table 1

Differencing Assembly (DA) Properties

\begin{tabular}{lccccccccc}
\hline \hline DA & $\begin{array}{c}\lambda^{\mathrm{a}} \\
(\mathrm{mm})\end{array}$ & $\begin{array}{c}v^{\mathrm{a}} \\
(\mathrm{GHz})\end{array}$ & $g(v)^{\mathrm{b}}$ & $\begin{array}{c}\theta_{\mathrm{FWHM}}{ }^{\mathrm{c}} \\
\left.{ }^{\circ}\right)\end{array}$ & $\begin{array}{c}\sigma_{0}(\mathrm{I})^{\mathrm{d}} \\
(\mathrm{mK})\end{array}$ & $\begin{array}{c}\sigma_{0}(\mathrm{Q}, \mathrm{U})^{\mathrm{d}} \\
(\mathrm{mK})\end{array}$ & $\begin{array}{c}v_{\mathrm{s}}^{\mathrm{e}} \\
(\mathrm{GHz})\end{array}$ & $\begin{array}{c}v_{\mathrm{ff}} \mathrm{e} \\
(\mathrm{GHz})\end{array}$ & $\begin{array}{c}v_{\mathrm{d}} \mathrm{e} \\
(\mathrm{GHz})\end{array}$ \\
\hline $\mathrm{K} 1$ & 13.17 & 22.77 & 1.0135 & 0.807 & 1.436 & 1.453 & 22.47 & 22.52 & 22.78 \\
$\mathrm{Ka} 1$ & 9.079 & 33.02 & 1.0285 & 0.624 & 1.470 & 1.488 & 32.71 & 32.76 & 33.02 \\
$\mathrm{Q} 1$ & 7.342 & 40.83 & 1.0440 & 0.480 & 2.254 & 2.278 & 40.47 & 40.53 & 40.85 \\
$\mathrm{Q} 2$ & 7.382 & 40.61 & 1.0435 & 0.475 & 2.141 & 2.163 & 40.27 & 40.32 & 40.62 \\
$\mathrm{~V} 1$ & 4.974 & 60.27 & 1.0980 & 0.324 & 3.314 & 3.341 & 59.65 & 59.74 & 60.29 \\
$\mathrm{~V} 2$ & 4.895 & 61.24 & 1.1010 & 0.328 & 2.953 & 2.975 & 60.60 & 60.70 & 61.27 \\
$\mathrm{~W} 1$ & 3.207 & 93.49 & 1.2480 & 0.213 & 5.899 & 5.929 & 92.68 & 92.82 & 93.59 \\
$\mathrm{~W} 2$ & 3.191 & 93.96 & 1.2505 & 0.196 & 6.565 & 6.602 & 93.34 & 93.44 & 94.03 \\
$\mathrm{~W} 3$ & 3.226 & 92.92 & 1.2445 & 0.196 & 6.926 & 6.964 & 92.34 & 92.44 & 92.98 \\
$\mathrm{~W} 4$ & 3.197 & 93.76 & 1.2495 & 0.210 & 6.761 & 6.800 & 93.04 & 93.17 & 93.84 \\
\hline
\end{tabular}

Notes.

${ }^{a}$ Effective wavelength and frequency for a thermodynamic spectrum.

b Conversion from antenna temperature to thermodynamic temperature, $\Delta T=$ $g(v) \Delta T_{A}$.

c Full-width-at-half-maximum from radial profile of A- and B-side average beams. Note: beams are not Gaussian.

d Noise per observation for resolution 9 and $10 \mathrm{I}, Q, \& U$ maps, to $\sim 0.1 \%$ uncertainty. $\sigma(p)=\sigma_{0} N_{\mathrm{obs}}^{-1 / 2}(p)$.

e Effective frequency for synchrotron (s), free-free (ff), and dust (d) emission, assuming spectral indices of $\beta=-2.9,-2.1,+2.0$, respectively, in antenna temperature units.

designated 1 or 2 (e.g., V11 or W12) depending on polarization mode.

In this paper, we follow the notation convention that flux density is $S \sim v^{\alpha}$ and antenna temperature is $T \sim v^{\beta}$, where the spectral indices are related by $\beta=\alpha-2$. In general, the CMB is expressed in terms of thermodynamic temperature, while Galactic and extragalactic foregrounds are expressed in antenna temperature. Thermodynamic temperature differences are given by $\Delta T=\Delta T_{A}\left[\left(e^{x}-1\right)^{2} / x^{2} e^{x}\right]$, where $x=h v / k T_{0}, h$ is the Planck constant, $v$ is the frequency, $k$ is the Boltzmann constant, and $T_{0}=2.725 \mathrm{~K}$ is the CMB temperature (Mather et al. 1999). A WMAP band-by-band tabulation of the conversion factors between thermodynamic and antenna temperature is given in Table 1.

\section{CHANGES IN THE FIVE-YEAR DATA ANALYSIS}

The one-year and three-year data analyses were described in detail in previous papers. In large part, the five-year analysis employs the same methods, so we do not repeat a detailed processing description here. However, we have made several improvements that are summarized here and described in more detail later in this paper and in a series of companion papers, as noted. We list the changes in order.

1. There is a $\sim 1^{\prime}$ temperature-dependent pointing offset between the star tracker coordinate system (which defines spacecraft coordinates) and the instrument boresights. In the three-year analysis we introduced a correction to account for the elevation change of the instrument boresights in spacecraft coordinates. With additional years of data, we have been able to refine our thermal model of the pointing offset, so we now include a small $\left(<1^{\prime}\right)$ correction to account for the azimuth change of the instrument boresights. Details of the new correction are given in the five-year Explanatory Supplement (Limon et al. 2008).

2. We have critically re-examined the relative and absolute intensity calibration procedures, paying special attention to the absolute gain recovery obtainable from the modulation of the CMB dipole due to WMAP's motion. We describe the revised procedure in Section 4 and note that the sky map calibration uncertainty has decreased from $0.5 \%$ to $0.2 \%$.

3. The WMAP beam response has now been measured in ten independent "seasons" of Jupiter observations. In the highest resolution W-band channels, these measurements now probe the beam response $\sim 44 \mathrm{~dB}$ down from the beam peak. However, there is still non-negligible beam solid angle below this level $(\sim 0.5 \%)$ that needs to be measured to enable accurate cosmological inference. In the three-year analysis we produced a physical optics model of the A-side beam response starting with a pre-flight model and fitting in-flight mirror distortions to the flight Jupiter data. In the five-year analysis we have extended the model to the B-side optics and, for both sides, we have extended the fit to include distortion modes a factor of 2 smaller in linear scale (four times as many modes). The model is used to augment the flight beam maps below a given threshold. The details of this work are given in Hill et al. (2009).

4. The far-sidelobe response of the beam was determined from a combination of ground measurements and in-flight lunar data taken early in the mission (Barnes et al. 2003). For the current analysis, we have replaced a small fraction of the far-sidelobe data with the physical optics model described above. We have also made the following changes in our handling of the far-sidelobe pickup (Hill et al. 2009). (1) we have enlarged the "transition radius" that defines the boundary between the main-beam and the far-sidelobe responses. This places a larger fraction of the total beam solid angle in the main beam where uncertainties are easier to quantify and propagate into the angular power spectra. (2) We have moved the far-sidelobe deconvolution into the combined calibration and sky map solver (Section 4). This produces a self-consistent estimate of the intensity calibration and the deconvolved sky map. The calibrated time-ordered data archive has had an estimate of the farsidelobe response subtracted from each datum (as it had in the three-year processing).

5. We have updated the optimal filters used in the final step of map making. The functional form of the filter is unchanged (Jarosik et al. 2007), but the fits have been updated to cover years 4 and 5 of the flight data.

6. Each WMAP DA consists of two radiometers that are sensitive to orthogonal linear polarization states. The sum and difference of the two radiometer channels split the signal into intensity and polarization components, respectively. However, the noise levels in the two radiometers are not equal, in general, so more optimal sky map estimation is possible in theory, at the cost of mixing intensity and polarization components in the process. For the current analysis, we investigated one such weighted algorithm and found that the polarization maps were subject to unacceptable contamination by the intensity signal in cases where the beam response was non-circular and the gradient of the intensity signal was large, e.g., in the K-band data. As a result, we reverted to the unweighted (and unbiased) estimator used in previous work.

7. We have improved the sky masks used to reject foreground contamination. In previous work, we defined masks based on contours of the K-band data. In the five-year analysis we produce masks based jointly on K-band and Q-band contours. For a given sky cut fraction, the new masks exclude 
Table 2

Lost and Rejected Data

\begin{tabular}{lccccc}
\hline \hline Category & K Band & Ka Band & Q Band & V Band & W Band \\
\hline Lost or incomplete telemetry (\%) & 0.12 & 0.12 & 0.12 & 0.12 & 0.12 \\
Spacecraft anomalies (\%) & 0.44 & 0.46 & 0.52 & 0.44 & 0.48 \\
Planned station keeping maneuvers (\%) & 0.39 & 0.39 & 0.39 & 0.39 & 0.39 \\
Planet in beam (\%) & 0.11 & 0.11 & 0.11 & 0.11 & 0.11 \\
& $\ldots$ & $\ldots$ & $\ldots$ & $\ldots$ & $\ldots$ \\
Total lost or rejected $(\%)$ & 1.06 & 1.08 & 1.14 & 1.06 & 1.10 \\
\hline
\end{tabular}

flat spectrum (e.g., free-free) emission more effectively. The new masks are described in detail in Gold et al. (2009) and are provided with the five-year data release. In addition, we have modified the "processing" mask used to exclude very bright sources during sky map estimation. The new mask is defined in terms of low-resolution (r4) HEALPix sky pixels (Gorski et al. 2005) to facilitate a cleaner definition of the pixel-pixel inverse covariance matrices, $N^{-1}$. One side effect of this change is to introduce a few r4-sized holes around the brightest radio sources in the analysis mask, which incorporates the processing mask as a subset.

8. We have amended our foreground analysis in the following ways: (1) Gold et al. (2009) perform a pixel-by-pixel analysis of the joint temperature and polarization data to study the breakdown of the Galactic emission into physical components. (2) We have updated some aspects of the Maximum Entropy (MEM) based analysis, as described in Gold et al. (2009). (3) Dunkley et al. (2009) develop a new analysis of polarized foreground emission using a Gibbs sampling approach that yields a cleaned CMB polarization map and an associated covariance matrix. (4) Wright et al. (2009) update the WMAP point source catalog and present some results on variable sources in the five-year data. However, the basic cosmological results are still based on maps that were cleaned with the same template-based procedure that was used in the three-year analysis.

9. We have improved the final temperature power spectrum, $C_{l}^{T T}$, by using a Gibbs-based maximum-likelihood estimate for $l \leqslant 32$ (Dunkley et al. 2009) and a pseudo- $C_{l}$ estimate for higher $l$ (Nolta et al. 2009). As with the threeyear analysis, the pseudo- $C_{l}$ estimate uses only $\mathrm{V}$ - and W-band data. With five individual years of data and six $\mathrm{V}$ - and W-band DAs, we can now form individual crosspower spectra from 15 DA pairs within each of five years and from 36 DA pairs across 10 year pairs, for a total of 435 independent cross-power spectra.

10. In the three year analysis we developed a pseudo- $C_{l}$ method for evaluating polarization power spectra in the presence of correlated noise. In the present analysis we additionally estimate the TE, TB, EE, EB, and BB spectra and their errors using an extension of the maximumlikelihood method in Page et al. (2007). However, as in the three-year analysis, the likelihood of a given model is still evaluated directly from the polarization maps using a pixel-based likelihood.

11. We have improved the form of the likelihood function used to infer cosmological parameters from the Monte Carlo Markov chains (Dunkley et al. 2009). We use an exact maximum-likelihood form for the $l \leqslant 32$ TT data (Eriksen et al. 2007b). We have investigated theoretically optimal methods for incorporating window function uncertainties into the likelihood, but in tests with simulated data we have found them to be biased. In the end, we adopt the form used in the three-year analysis (Hinshaw et al. 2007), but we incorporate the smaller five-year window function uncertainties (Hill et al. 2009) as inputs. We now routinely account for gravitational lensing when assessing parameters, and we have added an option to use low- $l$ TB and EB data for testing non-standard cosmological models.

12. For testing non-Gaussianity, we employ an improved estimator for $f_{\mathrm{NL}}$ (Creminelli et al. 2006; Yadav et al. 2007). The results of this analysis are described in Komatsu et al. (2009).

\section{OBSERVATIONS AND MAPS}

The five-year WMAP data encompass the period from 00:00:00 UT, 2001 August 10 (day number 222) to 00:00:00 UT, 2006 August 9 (day number 222). The observing efficiency during this time is roughly $99 \%$; Table 2 lists the fraction of data that were lost or rejected as unusable. The Table also gives the fraction of data that are flagged due to potential contamination by thermal emission from Mars, Jupiter, Saturn, Uranus, and Neptune. These data are not used in map making, but are useful for in-flight beam mapping (Hill et al. 2009; Limon et al. 2008).

After performing an end-to-end analysis of the instrument calibration, single-year sky maps are created from the timeordered data using the procedure described by Jarosik et al. (2007). Figure 1 shows the five-year temperature maps at each of the five WMAP observing frequencies: 23, 33, 41, 61, and $94 \mathrm{GHz}$. The number of independent observations per pixel, $N_{\text {obs }}$, is qualitatively the same as Figure 2 of Hinshaw et al. (2007) and is not reproduced here. The noise per pixel, $p$, is given by $\sigma(p)=\sigma_{0} N_{\mathrm{obs}}^{-1 / 2}(p)$, where $\sigma_{0}$ is the noise per observation, given in Table 1 . To a very good approximation, the noise per pixel in the five-year maps is a factor of $\sqrt{5}$ times lower than in the single-year maps. Figures 2 and 3 show the five-year polarization maps in the form of the Stokes parameters $Q$ and $U$, respectively. Maps of the relative polarization sensitivity, the $Q$ and $U$ analogs of $N_{\mathrm{obs}}$, are shown in Figure 13 of Jarosik et al. (2007) and are not updated here. A description of the low-resolution pixel-pixel inverse covariance matrices used in the polarization analysis is also given in Jarosik et al. (2007), and is not repeated here. The polarization maps are dominated by foreground emission, primarily synchrotron emission from the Milky Way. Figure 4 shows the polarization maps in a form in which the color scale represents polarized intensity, $P=\sqrt{Q^{2}+U^{2}}$, and the line segments indicate polarization direction for pixels with a signal-to-noise ratio greater than 1 . As with the temperature maps, the noise per pixel in the fiveyear polarization maps is $\sqrt{5}$ times lower than in the single-year maps.

Figure 5 shows the difference between the five-year temperature maps and the corresponding three-year maps. All maps have been smoothed to $2^{\circ}$ resolution to minimize the noise 

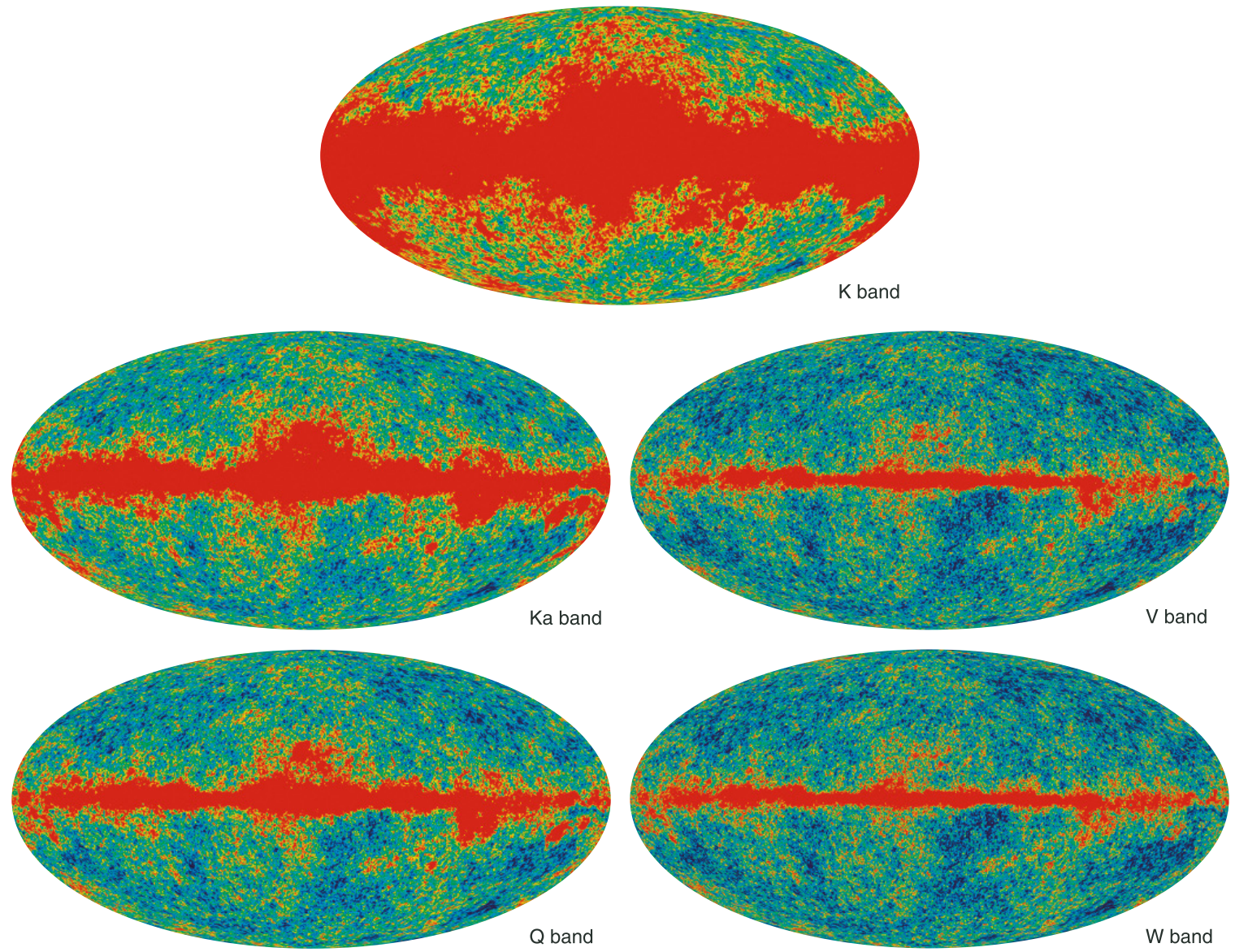

$-200$

$\mathrm{T}(\mu \mathrm{K})$

$+200$

Figure 1. Five-year temperature sky maps in Galactic coordinates smoothed with a 0.2 Gaussian beam, shown in Mollweide projection. Top: $\mathrm{K}$ band (23 GHz), middle-left: Ka band $(33 \mathrm{GHz})$, bottom-left: Q band $(41 \mathrm{GHz})$, middle-right: V band $(61 \mathrm{GHz})$, and bottom-right: W band $(94 \mathrm{GHz})$.

Table 3

Change in Low- $l$ Power from Three-Year Data

\begin{tabular}{lcccc}
\hline \hline Band & $\begin{array}{c}l=0^{\mathrm{a}} \\
(\mu \mathrm{K})\end{array}$ & $\begin{array}{c}l=1^{\mathrm{a}} \\
(\mu \mathrm{K})\end{array}$ & $\begin{array}{c}l=2^{\mathrm{b}} \\
\left(\mu \mathrm{K}^{2}\right)\end{array}$ & $\begin{array}{c}l=3^{\mathrm{b}} \\
\left(\mu \mathrm{K}^{2}\right)\end{array}$ \\
\hline $\mathrm{K}$ & 9.3 & 5.1 & 4.1 & 0.7 \\
$\mathrm{Ka}$ & 18.9 & 2.1 & 2.8 & 0.2 \\
$\mathrm{Q}$ & 18.3 & 0.4 & 2.5 & 0.5 \\
$\mathrm{~V}$ & 14.4 & 7.3 & 1.2 & 0.0 \\
$\mathrm{~W}$ & 16.4 & 3.5 & 1.0 & 0.0 \\
\hline
\end{tabular}

Notes.

a $l=0,1$ - amplitude in the difference map, outside the processing cut, in $\mu \mathrm{K}$.

b $l=2,3$-power in the difference map, outside the processing cut, $l(l+$ 1) $C_{l} / 2 \pi$, in $\mu \mathrm{K}^{2}$.

difference between them (due to the additional years of data). The left column shows the difference without any further processing, save for the subtraction of a relative offset between the maps. Table 3 gives the value of the relative offset in each band. Recall that WMAP is insensitive to absolute temperature, so we adopt a convention that sets the zero level in each map based on a model of the foreground emission at the galactic poles. While we have not changed conventions, our three-year estimate was erroneous due to the use of a preliminary CMB signal map at the time the estimate was made. This error did not affect any cosmological results, but it probably explains the offset differences noted by Eriksen et al. (2008) in their recent analysis of the three-year data.
The dominant structure in the left column of Figure 5 consists of a residual dipole and galactic plane emission. This reflects the updated five-year calibration which has produced changes in the gain of order $0.3 \%$ compared to the three-year gain estimate (see Section 4 for a more detailed discussion of the calibration). Table 3 gives the dipole amplitude difference in each band, along with the much smaller quadrupole and octupole power difference (for comparison, we estimate the CMB power at $l=2,3$ to be $l(l+1) C_{l} / 2 \pi=211,1041 \mu \mathrm{K}^{2}$, respectively). The right column of Figure 5 shows the corresponding sky map differences after the three-year map has been rescaled by a single factor (in each band) to account for the mean gain change between the three and five-year calibration determinations. The residual galactic plane structure in these maps is less than $0.2 \%$ of the nominal signal in the $\mathrm{Q}$ band, and less than $0.1 \%$ in all the other bands. The large-scale structure in the band-averaged temperature maps is quite robust.

\subsection{CMB Dipole}

The dipole anisotropy stands apart from the rest of the CMB signal due to its large amplitude and to the understanding that it arises from our peculiar motion with respect to the $\mathrm{CMB}$ rest frame. In this section we present CMB dipole results based on a new analysis of the five-year sky maps. Aside from an absolute calibration uncertainty of $0.2 \%$ (see Section 4), the dominant source of uncertainty in the dipole estimate arises from uncertainties in Galactic foreground subtraction. Here we 

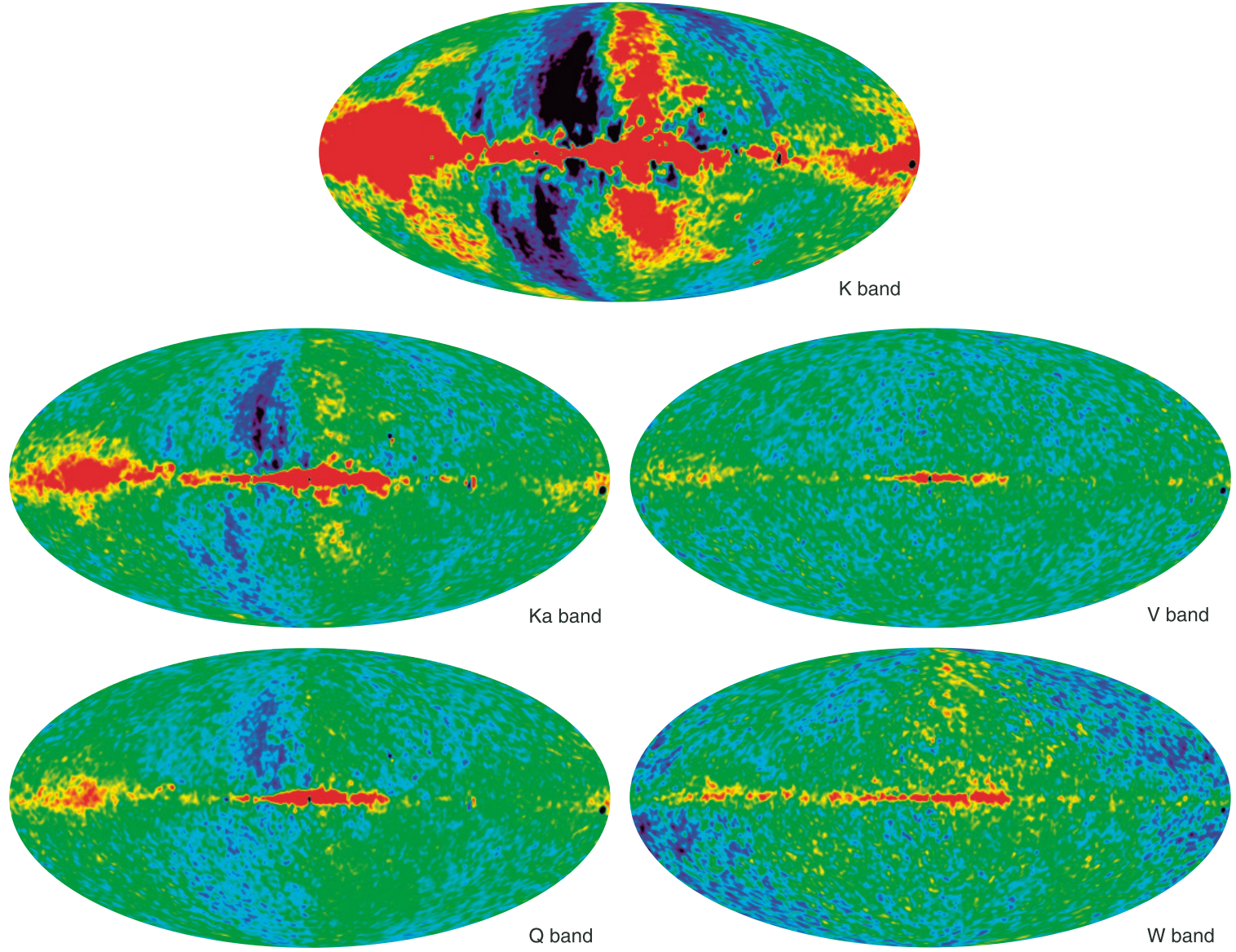

$-30$

$\mathrm{T}(\mu \mathrm{K})$

$+30$

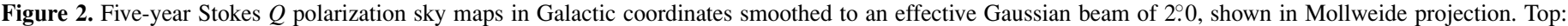
$\mathrm{K}$ band (23 GHz), middle-left: Ka band ( $33 \mathrm{GHz})$, bottom-left: Q band (41 GHz), middle-right: V band (61 GHz), and bottom-right: W band ( $94 \mathrm{GHz})$.

present results for two different removal methods: templatebased cleaning and an internal linear combination (ILC) of the WMAP multifrequency data (Gold et al. 2009). Our final results are based on a combination of these methods with uncertainties that encompass both approaches.

With template-based foreground removal, we can form cleaned maps for each of the eight high-frequency DAs, Q1$\mathrm{W} 4$, while the ILC method produces one cleaned map from a linear combination of all the WMAP frequency bands. We analyze the residual dipole moment in each of these maps (a nominal dipole based on the three-year data is subtracted from the time-ordered data prior to map making) using a Gibbs sampling technique which generates an ensemble of full-sky CMB realizations that are consistent with the data, as detailed below. We evaluate the dipole moment of each full-sky realization and compute uncertainties from the scatter of the realizations.

We prepared the data for the Gibbs analysis as follows. The $N_{\text {side }}=512$, template-cleaned maps were zeroed within the KQ85 mask, smoothed with a $10^{\circ}$ FWHM Gaussian kernel, and degraded to $N_{\text {side }}=16$. Zeroing the masked region prior to smoothing prevents residual cleaning errors within the mask from contaminating the unmasked data. We add random white noise (12 $\mu \mathrm{K} \mathrm{rms}$ per pixel) to each map to regularize the pixelpixel covariance matrix. The $N_{\text {side }}=512$ ILC map was also smoothed with a $10^{\circ}$ FWHM Gaussian kernel and degraded to $N_{\text {side }}=16$, but the data within the sky mask were not zeroed prior to smoothing. We add white noise of $6 \mu \mathrm{K}$ per pixel to the smoothed ILC map to regularize its covariance matrix. Note that smoothing the data with a $10^{\circ}$ kernel reduces the residual dipole in the maps by $\sim 0.5 \%$. We ignore this effect since the residual dipole is only $\sim 0.3 \%$ of the full dipole amplitude to start with.

The Gibbs sampler was run for 10,000 steps for each of the eight template-cleaned maps (Q1-W4) and for each of six independent noise realizations added to the ILC map. In both cases we applied the KQ85 mask to the analysis and truncated the CMB power at $l_{\max }=32$. The resulting ensembles of 80,000 and 60,000 dipole samples were analyzed independently and jointly. The results of this analysis are given in Table 4 . The first row combines the results from the template-cleaned DA maps; the scatter among the eight DAs was well within the noise scatter for each DA, so the Gibbs samples for all eight DAs were combined for this analysis. The results for the ILC map are shown in the second row. The two methods give reasonably consistent results, however, the Galactic longitude of the two dipole axis estimates differ from each other by about $2 \sigma$. Since we cannot reliably identify one cleaning method to be superior to the other, we have merged the Gibbs samples from both methods to produce the conservative estimate shown in the bottom row. This approach, which enlarges the uncertainty to encompass both estimates, gives

$$
\begin{aligned}
(d, l, b)= & (3.355 \pm 0.008 \mathrm{mK}, 263.99 \pm 0.14, \\
& 48.26 \pm 0.03),
\end{aligned}
$$



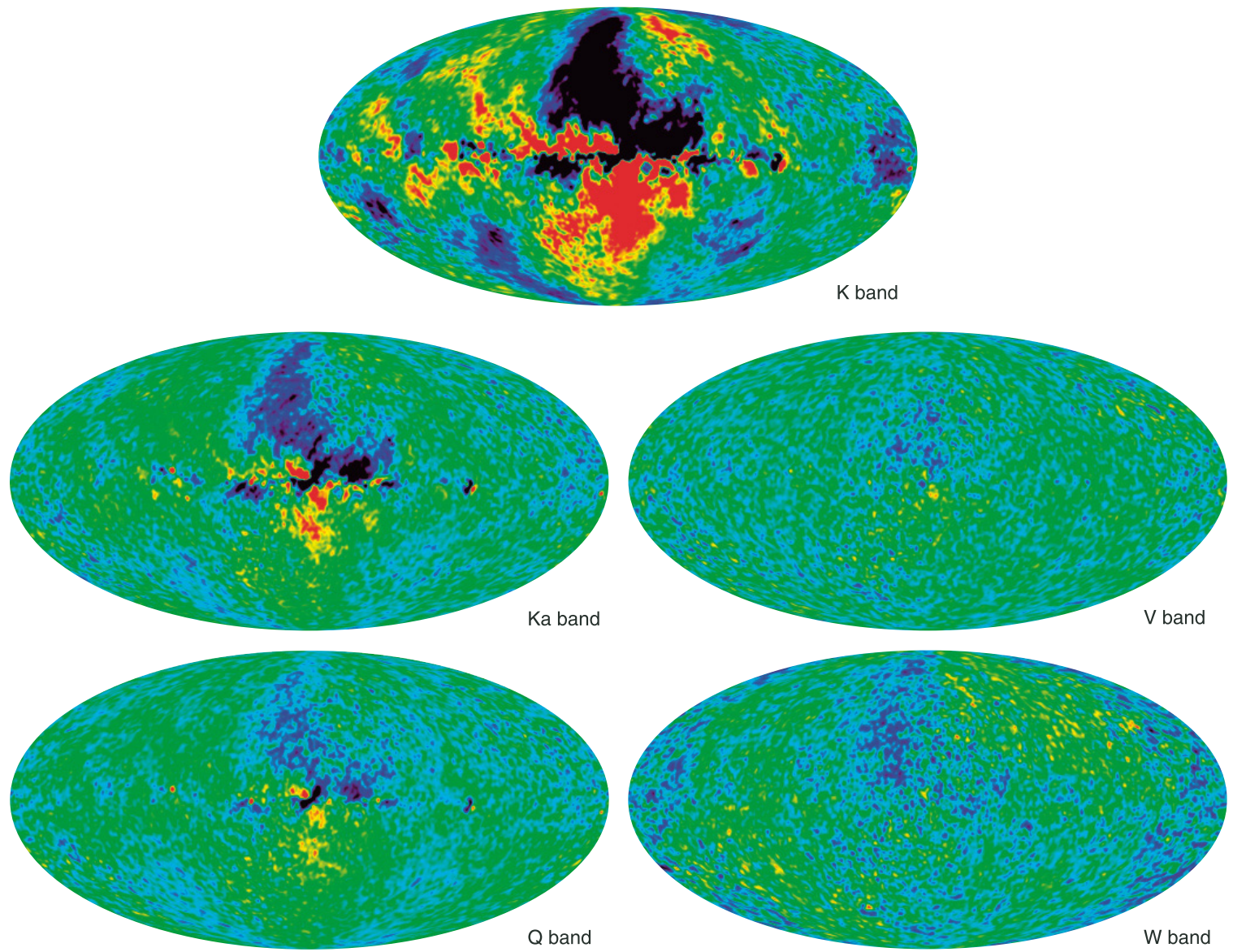

$-30$

$\mathrm{T}(\mu \mathrm{K})$

$+30$

Figure 3. Five-year Stokes $U$ polarization sky maps in Galactic coordinates smoothed to an effective Gaussian beam of 2.0 , shown in Mollweide projection. Top: $\mathrm{K}$ band $(23 \mathrm{GHz})$, middle-left: Ka band $(33 \mathrm{GHz})$, bottom-left: $\mathrm{Q}$ band $(41 \mathrm{GHz})$, middle-right: $\mathrm{V}$ band $(61 \mathrm{GHz})$, and bottom-right: W band (94 GHz).

where the amplitude estimate includes the $0.2 \%$ absolute calibration uncertainty. Given the CMB monopole temperature of $2.725 \mathrm{~K}$ (Mather et al. 1999), this amplitude implies a Solar System peculiar velocity of $369.0 \pm 0.9 \mathrm{~km} \mathrm{~s}^{-1}$ with respect to the CMB rest frame.

\section{CALIBRATION IMPROVEMENTS}

With the five-year processing we have refined our procedure for evaluating the instrument calibration, and have improved our estimates for the calibration uncertainty. The fundamental calibration source is still the dipole anisotropy induced by WMAP's motion with respect to the CMB rest frame (Hinshaw et al. 2003; Jarosik et al. 2007), but several details of the calibration fitting have been modified. The new calibration solution is consistent with previous results in the overlapping time range. We estimate the uncertainty in the absolute calibration is now $0.2 \%$ per DA.

The basic calibration procedure posits that a single channel of time-ordered data, $d_{i}$, may be modeled as

$$
d_{i}=g_{i}\left[\Delta T_{v i}+\Delta T_{a i}\right]+b_{i},
$$

where $i$ is a time index, $g_{i}$ and $b_{i}$ are the instrument gain and baseline, at time step $i, \Delta T_{v i}$ is the differential dipole anisotropy induced by WMAP's motion, and $\Delta T_{a i}$ is the differential sky anisotropy. We assume that $\Delta T_{v i}$ is known exactly and has the form

$$
\Delta T_{v i}=\frac{T_{0}}{c} \mathbf{v}_{i} \cdot\left[\left(1+x_{\mathrm{im}}\right) \mathbf{n}_{A, i}-\left(1-x_{\mathrm{im}}\right) \mathbf{n}_{B, i}\right],
$$

where $T_{0}=2.725 \mathrm{~K}$ is the CMB temperature (Mather et al. 1999), $c$ is the speed of light, $\mathbf{v}_{i}$ is WMAP's velocity with respect to the CMB rest frame at time step $i, x_{\mathrm{im}}$ is the loss imbalance parameter (Jarosik et al. 2007), and $\mathbf{n}_{A, i}$, and $\mathbf{n}_{B, i}$ are the unit vectors of the A- and B-side lines of sight at time step $i$ (in the same frame as the velocity vector). The velocity may be decomposed as

$$
\mathbf{v}_{i}=\mathbf{v}_{\mathrm{WMAP}-\mathrm{SSB}, \mathrm{i}}+\mathbf{v}_{\mathrm{SSB}-\mathrm{CMB}},
$$

where the first term is WMAP's velocity with respect to the solar system barycenter, and the second is the barycenter velocity with respect to the CMB. The former is well determined from ephemeris data, while the latter has been measured by COBEDMR with an uncertainty of $0.7 \%$ (Kogut et al. 1996). Since the latter velocity is constant over WMAP's life span, any error in our assumed value of $\mathbf{v}_{\mathrm{SSB}-\mathrm{CMB}}$ will, in theory, be absorbed into a dipole contribution to the anisotropy map, $T_{a}$. We test this hypothesis below. The differential sky signal has the form

$$
\begin{aligned}
\Delta T_{a i}= & \left(1+x_{\mathrm{im}}\right)\left[I_{a}\left(p_{A, i}\right)+P_{a}\left(p_{A, i}, \gamma_{A, i}\right)\right]-\left(1-x_{\mathrm{im}}\right) \\
& \times\left[I_{a}\left(p_{B, i}\right)+P_{a}\left(p_{B, i}, \gamma_{B, i}\right)\right],
\end{aligned}
$$



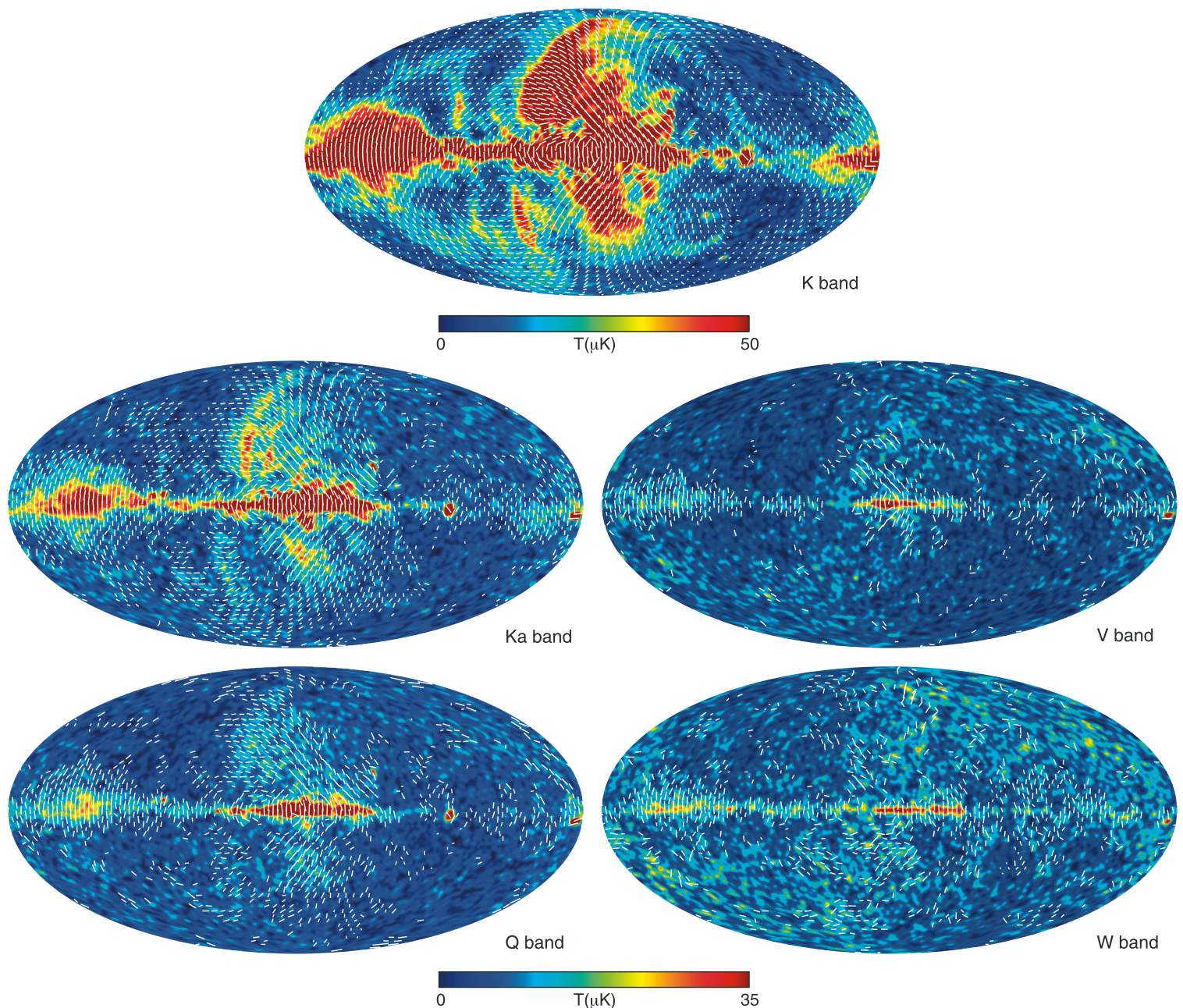

Figure 4. Five-year polarization sky maps in Galactic coordinates smoothed to an effective Gaussian beam of 2.0, shown in Mollweide projection. The color scale indicates polarized intensity, $P=\sqrt{Q^{2}+U^{2}}$, and the line segments indicate polarization direction in pixels whose signal-to-noise exceeds 1 . Top: $\mathrm{K}$ band (23 GHz), middle-left: Ka band $(33 \mathrm{GHz})$, bottom-left: Q band $(41 \mathrm{GHz})$, middle-right: $\mathrm{V}$ band $(61 \mathrm{GHz})$, and bottom-right: $\mathrm{W}$ band $(94 \mathrm{GHz})$.

where $p_{A, i}$ is the pixel observed by the A-side at time step $i$ (and similarly for $\mathrm{B}), I_{a}(p)$ is the temperature anisotropy in pixel $p$ (the intensity Stokes parameter, $I)$, and $P_{a}(p, \gamma)$ is the polarization anisotropy in pixel $p$ at polarization angle $\gamma$ (Hinshaw et al. 2003) which is related to the linear Stokes parameters $Q$ and $U$ by

$$
P_{a}(p, \gamma)=Q(p) \cos 2 \gamma+U(p) \sin 2 \gamma .
$$

We further note that, in general, $I_{a}$ and $P_{a}$ depend on frequency owing to Galactic emission.

A main goal of the data processing is to simultaneously fit for the calibration and sky signal. Unfortunately, since the data model is nonlinear and the number of parameters is large, the general problem is intractable. In practice, we proceed iteratively as follows. Initially we assume that the gain and baseline are constant for a given time interval, typically between 1 and $24 \mathrm{~h}$,

$$
\begin{array}{cl}
g_{i}=G_{k} & \tau_{k}<t_{i}<\tau_{k+1} \\
b_{i}=B_{k} & \tau_{k}<t_{i}<\tau_{k+1},
\end{array}
$$

where $t_{i}$ is the time of the $i$ th individual observation, and $\tau_{k}$ is the start time of the $k$ th calibration interval. Throughout the fit we fix the velocity-induced signal, Equation (3), using
$\mathbf{v}_{\mathrm{SSB}-\mathrm{CMB}}=[-26.29,-244.96,+275.93] \mathrm{km} \mathrm{s}^{-1}$ (in Galactic coordinates), and, for the first iteration, we assume no anisotropy signal, $\Delta T_{a}=0$. Then, for each calibration interval $k$ we perform a linear fit for $G_{k}$ and $B_{k}$ with fixed $\Delta T_{v}+\Delta T_{a}$. As we proceed through the intervals, we apply this calibration to the raw data and accumulate a new estimate of the anisotropy map as per Equation (19) of Hinshaw et al. (2003). The procedure is repeated with each updated estimate of $\Delta T_{a}$. Once the calibration solution has converged, we fit the gain data, $G_{k}$, to a model that is parameterized by the instrument detector voltage and the temperatures of the receiver's warm and cold stages, Equation (2) of Jarosik et al. (2007). This parametrization still provides a good fit to the $G_{k}$ data, so we have not updated its form for the five-year analysis. The updated best-fit parameters are given in the five-year Explanatory Supplement (Limon et al. 2008). Note that for each radiometer, the relative gain versus time over five years is determined by just two parameters.

For the five-year processing we have focused on the veracity of the "raw" calibration, $G_{k}$ and $B_{k}$. Specifically, we have improved and/or critically reexamined several aspects of the iterative fitting procedure:

1. We have incorporated the effect of far-sidelobe pickup directly into the iterative calibration procedure, rather than 

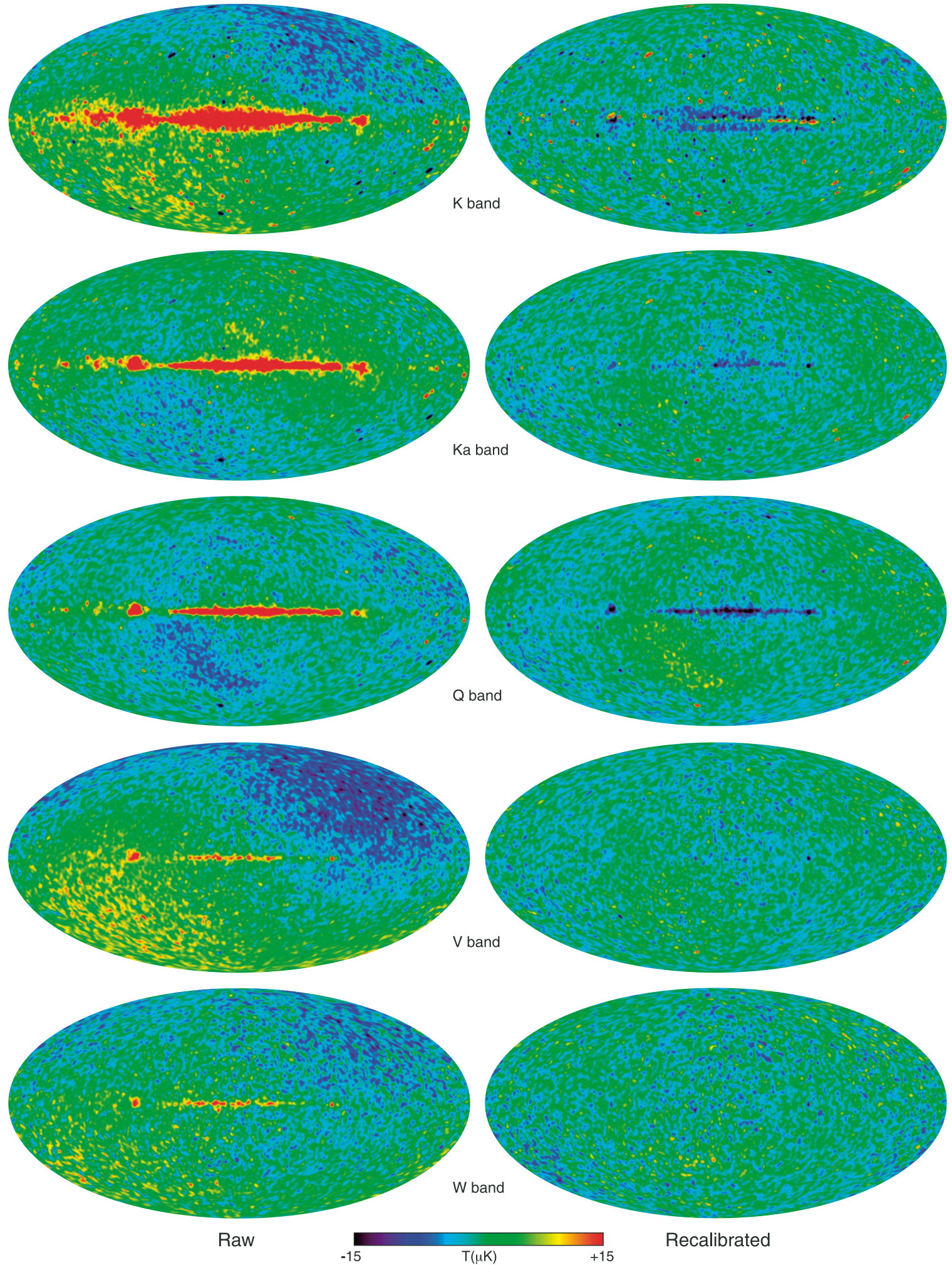

Figure 5. Difference between the five-year and three-year temperature maps. Left column: the difference in the maps, as delivered, save for the subtraction of a relative offset (Table 3), right column: the difference after correcting the three-year maps by a scale factor that accounts for the mean gain change, $\sim 0.3 \%$, between the three-year and five-year estimates. Top to bottom: K, Ka, Q, V, W bands. The differences before recalibration are dominated by galactic plane emission and a dipole residual: see Table 3, which also gives the changes for $l=2,3$.

as a fixed correction (Jarosik et al. 2007). We do this by segregating the differential signal into a main-beam contribution and a sidelobe contribution,

$$
\Delta T_{i}=\Delta T_{\text {main }, \mathrm{i}}+\Delta T_{\text {side }, \mathrm{i}} .
$$

(Hill et al. 2009 discuss how this segregation is defined in the five-year processing.) After each iteration of the calibration and sky map estimation, we (re)compute a database of $\Delta T_{\text {side }}$ on a grid of pointings using the new estimate of $I_{a}$. We then interpolate the database to estimate 
Table 4

WMAP Five-Year CMB Dipole Anisotropy ${ }^{\mathrm{a}}$

\begin{tabular}{lcccccc}
\hline \hline $\begin{array}{l}\text { Cleaning } \\
\text { Method }\end{array}$ & $\begin{array}{c}d_{x}^{\mathrm{b}} \\
(\mathrm{mK})\end{array}$ & $\begin{array}{c}d_{y} \\
(\mathrm{mK})\end{array}$ & $\begin{array}{c}d_{z} \\
(\mathrm{mK})\end{array}$ & $\begin{array}{c}d^{\mathrm{c}} \\
(\mathrm{mK})\end{array}$ & $\begin{array}{c}l \\
\left({ }^{\circ}\right)\end{array}$ & $\begin{array}{c}b \\
\left({ }^{\circ}\right)\end{array}$ \\
\hline Templates & $-0.229 \pm 0.003$ & $-2.225 \pm 0.003$ & $2.506 \pm 0.003$ & $3.359 \pm 0.008$ & $264.11 \pm 0.08$ & $48.25 \pm 0.03$ \\
ILC & $-0.238 \pm 0.003$ & $-2.218 \pm 0.002$ & $2.501 \pm 0.001$ & $3.352 \pm 0.007$ & $263.87 \pm 0.07$ & $48.26 \pm 0.02$ \\
Combined & $-0.233 \pm 0.005$ & $-2.222 \pm 0.004$ & $2.504 \pm 0.003$ & $3.355 \pm 0.008$ & $263.99 \pm 0.14$ & $48.26 \pm 0.03$
\end{tabular}

Notes.

${ }^{a}$ The CMB dipole components for two different galactic cleaning methods are given in the first two rows. The Gibbs samples from each set are combined in the last row to produce an estimate with conservative uncertainties that encompasses both cases.

$\mathrm{b}$ The Cartesian dipole components are given in Galactic coordinates. The quoted uncertainties reflect the effects of noise and sky cut, for illustration. An absolute calibration uncertainty of $0.2 \%$ should be added in quadrature.

${ }^{\mathrm{c}}$ The spherical components of the dipole are given in Galactic coordinates. In this case the quoted uncertainty in the magnitude, $d$, includes the absolute calibration uncertainty.

$\Delta T_{\text {side, } i}$ for each time step $i$. Note that $\Delta T_{\text {side }}$ includes contributions from both the velocity-induced signal and the intrinsic anisotropy. Ignoring sidelobe pickup can induce gain errors of up to $1.5 \%$ in the $\mathrm{K}$ band, $0.4 \%$ in the $\mathrm{Ka}$ band, and $\sim 0.25 \%$ in the $\mathrm{Q}-\mathrm{W}$ bands.

2. In general, the different channels within a DA have different center frequencies (Jarosik et al. 2003); hence the different channels measure a slightly different anisotropy signal due to differences in the Galactic signal. We assess the importance of accounting for this in the calibration procedure.

3. A single DA channel is only sensitive to a single linear polarization state. (WMAP measures polarization by differencing orthogonal polarization channels.) Thus we cannot reliably solve for both $P_{a}$ and for $I_{a}$ at each channel's center frequency. We assess the relative importance of accounting for one or the other on both the gain and baseline solutions.

4. We examine the sensitivity of the calibration solution to the choice of $\mathbf{v}_{\text {SSB-CMB }}$ and to assumptions of time dependence in the gain.

\subsection{Calibration Tests}

We use a variety of end-to-end simulations to assess and control the systematic effects noted above. We summarize a number of key tests in the remainder of this section.

The first case we consider is a noiseless simulation in which we generate time-ordered data from an input anisotropy map which includes CMB and Galactic foreground signal (one map per channel, evaluated at the center frequency of each channel) and a known dipole amplitude. The input gain for each channel is fixed to be constant in time. We run the iterative calibration and sky map solver allowing for an independent sky map solution at each channel (but no polarization signal). When fitting for the calibration, we assume that $\mathbf{v}_{\text {SSB-CMB }}$ differs from the input value by $1 \%$ to see if the known, modulated velocity term, $\mathbf{v}_{\text {WMAP-SSB }}$, properly "anchors" the absolute gain solution. The results are shown in the top panel of Figure 6 where it is shown that the absolute gain recovery is robust to errors in $\mathbf{v}_{\mathrm{SSB}-\mathrm{CMB}}$. We recover the input gain to better than $0.1 \%$ in this instance.

The second case we consider is again a noiseless simulation that now includes only dipole signal (with Earth-velocity modulation), but here we vary the input gain using the flight-derived gain model (Jarosik et al. 2007). The iterative solver was run on the K-band data for 1400 iterations, again starting with an initial guess that was in error by $1 \%$. The results are shown in the bottom panel of Figure 6, which indicate systematic convergence errors of $>0.3 \%$ in the fitted amplitude of the recovered gain model. Since the input sky signal in this case does not
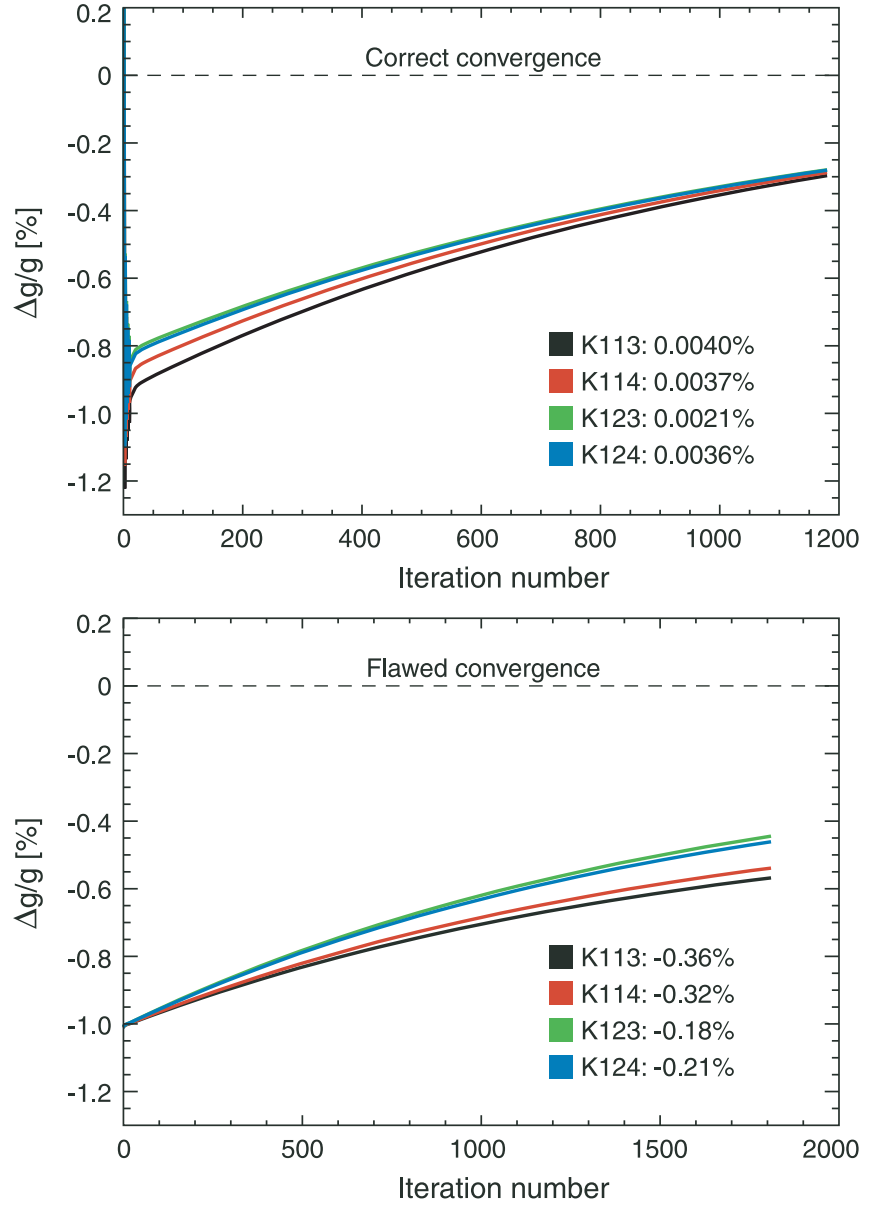

Figure 6. Gain convergence tests using the iterative sky map \& calibration solver run on a pair of simulations with known, but different, inputs. Both panels show the recovered gain as a function of iteration number for a four-channel K-band simulation. The initial calibration guess was chosen to be in error by $1 \%$ to test convergence; the output solutions, extrapolated with an exponential fit, are printed in each panel. Top: the results for a noiseless simulation that includes a dipole signal (with Earth-velocity modulation) plus CMB and foreground anisotropy (the former is evaluated at the center frequency of each channel). The input gain was set to be constant in time. The extrapolated solutions agree with the input values to much better than $0.1 \%$. Bottom: the results for a noiseless simulation that includes only dipole signal (with Earth-velocity modulation) but no CMB or foreground signal. In this case the input gain was set up to have flight-like thermal variations. The extrapolated absolute gain recovery was in error by $>0.3 \%$, indicating a small residual degeneracy between the sky model and the time-dependent calibration.

have any Galactic foreground or polarization components, we cannot ascribe the recovery errors to the improper handling of 


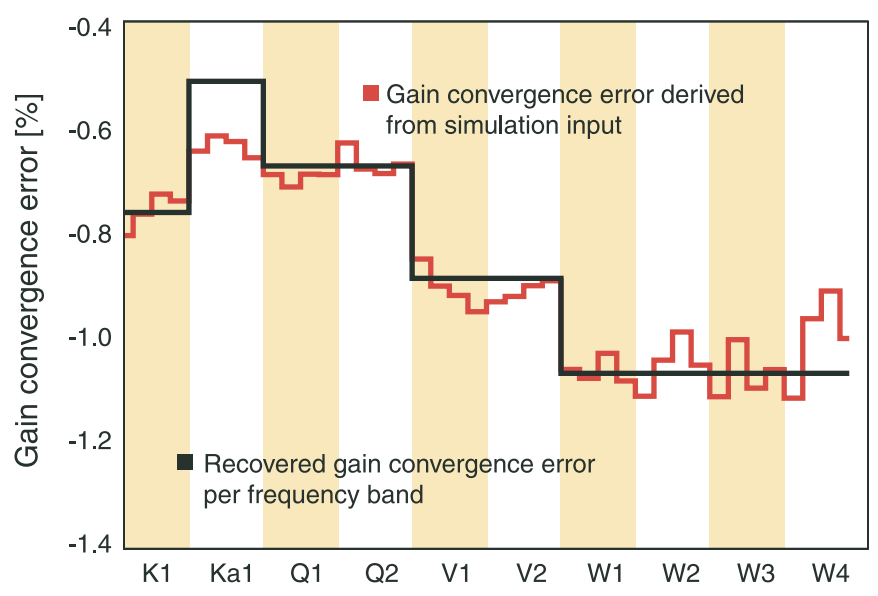

Figure 7. The gain error recovery test from a flight-like simulation that includes every effect known to be important. Using the daily dipole gains recovered from the iterative sky map and calibration solver as input, the gain convergence error, shown here, is fitted simultaneously with the gain model parameters, not shown, following the procedure outlined in Appendix B. The red trace indicates the true gain error for each WMAP channel, based on the known input gain and the gain solution achieved by the iterative solver on its final iteration. The black trace shows the gain error recovered by the fit, averaged by the frequency band. The channel-to-channel scatter within a band is $<0.1 \%$, though the mean of the Ka-band error is of order $0.1 \%$.

those effects in the iterative solver. We have also run numerous other simulations that included various combinations of instrument noise, CMB anisotropy, Galactic foreground signal (with or without individual center frequencies per channel), polarization signal, and input gain variations. The combination of runs is too numerous to report on in detail, and the results are not especially enlightening. The most pertinent trend we can identify is that when the input value of $\mathbf{v}_{\mathrm{SSB}-\mathrm{CMB}}$ is assumed in the iterative solver, the recovered gain is in good agreement with the input, but when the initial guess is in error by $1 \%$, the recovered gain will have comparable errors. We believe the lack of convergence is due to a weak degeneracy between gain variations and the sky map solution. Such a degeneracy is difficult to diagnose in the context of this iterative solver, especially given the computational demands of the system, so we are assessing the system more directly with a low-resolution parameterization of the gain and sky signal, as outlined in Appendix A.

Since the latter effort is still underway, we have adopted a more pragmatic approach to evaluating the absolute gain and its uncertainty for the five-year data release. We proceed as follows: after 50 iterations of the calibration and sky map solver, the dominant errors in the gain and sky map solution are (1) a dipole in the sky map, and (2) a characteristic wave form that reflects a relative error between $\mathbf{v}_{\mathrm{SSB}-\mathrm{CMB}}$ and $\mathbf{v}_{\mathrm{WMAP}-\mathrm{SSB}}$. At this point we can calibrate the amplitude of the gain error wave form to the magnitude of the velocity error in $\mathbf{v}_{\mathrm{SSB}-\mathrm{CMB}}$. We can then fit the gain solution to a linear combination of the gain model of Jarosik et al. (2007) and the velocity error wave form. See Appendix B for details on this fitting procedure. In practice this fit is performed simultaneously on both channels of a radiometer since those channels share one gain model parameter. We have tested this procedure on a complete flight-like simulation that includes every important effect known, including input gain variations. The results of the gain recovery are shown in Figure 7, and based on this we conservatively assign an absolute calibration uncertainty of $0.2 \%$ per channel for the five-year WMAP archive.

\subsection{Summary}

The series of steps taken to arrive at the final five-year calibration are as follows:

1. Run the iterative calibration and sky map solver over the full five-year data set for 50 iterations, using $24 \mathrm{hr}$ calibration intervals. This run starts with $I_{a}=P_{a}=0$ and updates $I_{a}$ for each individual channel of data. $P_{a}$ is assumed to be 0 throughout this run. We keep the gain solution, $G_{k}$, from this run and discard the baseline solution.

2. Run the iterative calibration and sky map solver over the full five-year data set for 50 iterations, using $1 \mathrm{hr}$ calibration intervals. This run starts with $I_{a}=P_{a}=0$ and updates both using the intensity and polarization data in the two radiometers per DA, as per Appendix D of Hinshaw et al. (2003). We keep the baseline solution, $B_{k}$, from this run and discard the gain solution. Both of these runs incorporate the sidelobe correction as noted above.

3 . Fit the gain solution, $G_{k}$ simultaneously for the gain model and for an error in the velocity, $\Delta \mathbf{v}_{\mathrm{SSB}-\mathrm{CMB}}$, as described in Appendix B. This fit is performed on two channels per radiometer with the gain model parameter $T_{0}$ common to both channels.

4. We average the best-fit velocity error over all channels within a frequency band under the assumption that the dipole is the same in each of these channels. We then fix the velocity error to a single value per frequency band and refit the gain model parameters for each pair of radiometer channels.

Based on end-to-end simulations with flight-like noise, we estimate the absolute gain error per radiometer to be $0.2 \%$. We believe the limiting factor in this estimate is a weak degeneracy between thermal variations in the instrument gain, which are annually modulated, and annual variations induced by errors in $\mathbf{v}_{\mathrm{SSB}-\mathrm{CMB}}$. Since there is a small monotonic increase in the spacecraft temperature, additional years of data should allow improvements in our ability to separate these effects.

Once we have finalized the gain model, we form a calibrated time-ordered data archive using the gain model and the $1 \mathrm{~h}$ baseline estimates to calibrate the data. This archive also has a final estimate of the far-sidelobe pickup subtracted from each time-ordered data point. However, we opt not to subtract a dipole estimate from the archive at this stage in the processing.

\section{BEAM IMPROVEMENTS}

In addition to reassessing the calibration, the other major effort undertaken to improve the five-year data processing was to extend the physical optics model of the WMAP telescope based on flight measurements of Jupiter. This work is described in detail in Hill et al. (2009) so we only summarize the key results with an emphasis on their scientific implications. The basic aim of the work is to use the flight beam maps from all ten DAs to determine the in-flight distortion of the mirrors. This program was begun for the A-side mirror during the threeyear analysis; for the five-year analysis we have quadrupled the number of distortion modes we fit (probing distortion scales that are half the previous size), and we have developed a completely new and independent model of the B-side distortions, rather than assuming that they mirror the A-side distortions. We have also placed limits on smaller-scale distortions by comparing the predicted beam response at large angles to sidelobe data collected during WMAP's early observations of the Moon. 
Given the best-fit mirror model, we compute the model beam response for each DA and use it in conjunction with the flight data to constrain the faint tails of the beams, beyond $\sim 1^{\circ}$ from the beam peak. These tails are difficult to constrain with flight data alone because the Jupiter signal to noise ratio is low, but, due to their large areal extent they contain a nonnegligible fraction (up to $1 \%$ ) of the total beam solid angle. An accurate determination of the beam tail is required to properly measure the ratio of subdegree-scale power to larger-scale power in the diffuse CMB emission (and to accurately assign point source flux).

Figure 14 in Hill et al. (2009) compares the beam radial profiles used in the three-year and five-year analyses, while Figure 13 compares the $l$-space transfer functions derived from the Legendre transform of the radial profile. The important changes to note are the following.

1. In both analyses we split the beam response into mainbeam and far-sidelobe contributions. In the five-year analysis we have enlarged the radius at which this transition is made (Hill et al. 2009). In both cases, we correct the timeordered data for far-sidelobe pickup prior to making sky maps, while the main-beam contribution is only accounted for in the analysis of sky maps, e.g., in power spectrum deconvolution. As a result, the sky maps have a slightly different effective resolution which is most apparent in the $\mathrm{K}$-band, as in Figure 5. However, in each analysis, the derived transfer functions are appropriate for the corresponding sky maps.

2. In the three-year analysis, the main-beam profile was described by a Hermite polynomial expansion fit to the observations of Jupiter in the time-ordered data. This approach was numerically problematic in the five-year analysis due to the larger transition radius; as a result, we now simply co-add the time-ordered data into radial bins to obtain the profiles. In both cases, the underlying timeordered data are a hybrid archive consisting of flight data for points where the beam model predicts a value above a given contour, and model values for points below the contour (Hill et al. 2009). With the improved beam models and a new error analysis, we have adjusted these hybrid contours down slightly, with the result that we use proportionately more flight data (per year) in the new analysis. The radius at which the five-year profile becomes model dominated ( $>50 \%$ of the points in a bin) is indicated by dotted lines in Figure 14 of Hill et al. (2009).

3. The right column of Figure 14 in Hill et al. (2008) shows the fractional change in solid angle due to the updated profiles. The main point to note is the $\sim 1 \%$ increase in the $\mathrm{V} 2$ and the W-band channels, primarily arising in the bin from 1 to $2 \mathrm{deg}$ off the beam peak. As can be seen in Figure 3 of Hill et al. (2009), this is the angular range in which the new beam models produced the most change, owing to the incorporation of smaller distortion modes in the mirror model. The three-year analysis made use of the model in this angular range which, in hindsight, was suppressing up to $\sim 1 \%$ of the solid angle in the $\mathrm{V}$ - and $\mathrm{W}$-band beams. (The longer wavelength channels are less sensitive to distortions in this range, so the change in solid angle is smaller for the $\mathrm{K}-\mathrm{Q}$ bands.) In the five-year analysis, we use relatively more flight data in this regime, so we are less sensitive to any remaining model uncertainties. Hill et al. (2009) place limits on residual model errors and propagate those errors into the overall beam uncertainty.
4. Figure 13 in Hill et al. (2009) compares the beam transfer functions, $b_{l}$, derived by transforming the three-year and five-year radial profiles. To factor out the effect of changing the transition radius, the three-year profiles were extended to the five-year radius using the far-sidelobe data, for this comparison. Since the transfer functions are normalized to 1 at $l=1$, the change is restricted to high $l$. In the $\mathrm{V}$ and $\mathrm{W}$ bands, $b_{l}$ has decreased by $\sim 0.5 \%-1 \%$ due largely to the additional solid angle picked up in the 1-2 deg range. This amounts to $\mathrm{a} \sim 1 \sigma$ change in the functions, as indicated by the red curves in the figure.

The calibrated angular power spectrum is proportional to $1 / g^{2} b_{l}^{2}$, where $g$ is the mean gain and $b_{l}$ is the beam transfer function; thus the net effect of the change in gain and beam determinations is to increase the power spectrum by $\sim 0.5 \%$ at $l \lesssim 100$, and by $\sim 2.5 \%$ at high $l$. Nolta et al. (2009) give a detailed evaluation of the power spectrum while Dunkley et al. (2009) and Komatsu et al. (2009) discuss the implications for cosmology.

\section{LOW- $l$ POLARIZATION TESTS}

The three-year data release included the first measurement of microwave polarization over the full sky, in the form of Stokes $Q$ and $U$ maps in each of five bands. The analysis of WMAP polarization data is complicated by the fact that the instrument was not designed to be a true polarimeter; thus a number of systematic effects had to be understood prior to assigning reliable error estimates to the data. Page et al. (2007) presented the three-year polarization data in great detail. In this section we extend that analysis by considering some additional tests that were not covered in the three-year analysis. We note that all of the tests described in this section have been performed on the template-cleaned reduced-foreground maps except for the final test of the Ka-band data, described at the end of the section, which tests an alternative cleaning method.

\subsection{Year-to-Year Consistency Tests}

With five-years of data it is now possible to subject the data to more stringent consistency tests than was previously possible. In general, the number of independent cross-power spectra we can form within a band with $N_{d}$ DAs is $N_{d}\left(N_{d}-1\right) / 2 \times N_{y}+$ $N_{d}^{2} \times N_{y}\left(N_{y}-1\right) / 2$. With five years of data, this gives ten independent estimates each in the $\mathrm{K}$ and $\mathrm{Ka}$ bands, 45 each in the Q and V bands, and 190 in the $\mathrm{W}$ band. For cross power spectra of distinct band pairs, with $N_{d 1}$ and $N_{d 2}$ DAs in each band, the number is $N_{d 1} N_{d 2} \times N_{y}^{2}$. This gives 50 each in $\mathrm{KaQ}$ and $\mathrm{KaV}, 100$ each in $\mathrm{KaW}$ and QV, and 200 each in QW and VW. For comparison, the corresponding numbers are 3, 15, and 66 , and 18,36 , and 72 with three years of data.

We have evaluated these individual spectra from the five-year data and have assigned noise uncertainties to each estimate using the Fisher formalism described in Page et al. (2007). We subject the ensemble to an internal consistency test by computing the reduced $\chi^{2}$ of the data at each multipole $l$ within each band or band pair, under the hypothesis that the data at each multipole and band measure the same number from DA to DA and year to year. The results of this test are given in Table 5 for the foreground-cleaned $\mathrm{EE}, \mathrm{EB}$, and $\mathrm{BB}$ spectra from $l=2-10$ for all band pairs from KaKa to WW. There are several points to note in these results.

1. For $l \geqslant 6$, the most significant deviation from 1 in reduced $\chi^{2}$, in any spectrum or band, is 1.594 in the $l=7 \mathrm{BB}$ 
Table 5

Polarization $\chi^{2}$ Consistency Tests ${ }^{\mathrm{a}}$

\begin{tabular}{|c|c|c|c|c|c|c|c|c|c|c|}
\hline Multipole & $\begin{array}{c}\mathrm{KaKa} \\
(10)^{\mathrm{b}}\end{array}$ & $\begin{array}{c}\mathrm{KaQ} \\
(50) \\
\end{array}$ & $\begin{array}{l}\mathrm{KaV} \\
(50) \\
\end{array}$ & $\begin{array}{l}\text { KaW } \\
(100) \\
\end{array}$ & $\begin{array}{l}\text { QQ } \\
(45) \\
\end{array}$ & $\begin{array}{c}\text { QV } \\
(100)\end{array}$ & $\begin{array}{c}\text { QW } \\
(200)\end{array}$ & $\begin{array}{l}\text { VV } \\
(45) \\
\end{array}$ & $\begin{array}{c}\text { VW } \\
(200)\end{array}$ & $\begin{array}{l}\text { WW } \\
(190)\end{array}$ \\
\hline \multicolumn{11}{|l|}{ EE } \\
\hline 2 & 0.727 & 1.059 & 1.019 & 1.301 & 1.586 & 0.690 & 1.179 & 0.894 & 1.078 & 1.15 \\
\hline 3 & 1.373 & 0.994 & 1.683 & 1.355 & 1.092 & 1.614 & 1.325 & 1.005 & 1.386 & 1.51 \\
\hline 4 & 1.561 & 1.816 & 1.341 & 2.033 & 0.993 & 1.126 & 1.581 & 1.195 & 1.596 & 1.724 \\
\hline 5 & 0.914 & 1.313 & 1.062 & 1.275 & 1.631 & 1.052 & 1.155 & 0.589 & 0.881 & 1.252 \\
\hline 6 & 1.003 & 0.847 & 0.688 & 1.124 & 0.740 & 0.856 & 1.049 & 1.384 & 1.168 & 1.142 \\
\hline 7 & 0.600 & 0.671 & 0.689 & 0.936 & 0.936 & 0.780 & 0.864 & 0.900 & 1.064 & 1.015 \\
\hline 8 & 1.578 & 1.262 & 1.337 & 1.212 & 1.080 & 0.763 & 0.608 & 1.025 & 0.871 & 0.749 \\
\hline 9 & 0.760 & 0.710 & 0.891 & 0.820 & 0.582 & 0.726 & 0.651 & 0.791 & 0.821 & 0.795 \\
\hline 10 & 0.494 & 0.821 & 0.996 & 0.914 & 0.656 & 0.763 & 0.806 & 0.676 & 0.891 & 0.94 \\
\hline \multicolumn{11}{|l|}{ EB } \\
\hline 2 & 00 & 1.297 & 1.179 & 2.074 & 1.006 & 0.9 & 2.1 & 1. & 2.085 & 2.30 \\
\hline 3 & 0.719 & 1.599 & 0.651 & 2.182 & 1.295 & 0.986 & 2.739 & 1. & 3.276 & 3.157 \\
\hline 4 & 0.746 & 1.702 & 1.378 & 1.777 & 1.926 & 1.110 & 1.435 & 1.028 & 1.279 & 1.861 \\
\hline 5 & 1.161 & 0.948 & 0.945 & 1.003 & 1.149 & 1.232 & 1.468 & 0.699 & 1.122 & 1.516 \\
\hline 6 & 0.475 & 1.183 & 0.651 & 0.687 & 0.829 & 1.023 & 0.814 & 1.201 & 1.136 & 0.960 \\
\hline 7 & 1.014 & 1.007 & 0.829 & 0.700 & 0.817 & 0.759 & 1.112 & 0.616 & 0.802 & 1.233 \\
\hline 8 & 0.849 & 0.897 & 1.279 & 0.861 & 0.681 & 0.689 & 0.955 & 1.021 & 0.954 & 0.99 \\
\hline 9 & 0.743 & 0.734 & 1.007 & 1.112 & 0.820 & 0.798 & 0.686 & 0.882 & 0.808 & 0.824 \\
\hline 10 & 0.413 & 1.003 & 1.316 & 0.859 & 0.722 & 0.900 & 0.693 & 1.124 & 0.836 & 0.852 \\
\hline \multicolumn{11}{|l|}{ BB } \\
\hline 2 & 38 & 1.570 & 4 & 2.497 & & & 2.5 & 0.6 & 1.631 & 9.19 \\
\hline 3 & 756 & 0.868 & 0.808 & 1.817 & 3.027 & 1.7 & 3.496 & 0.601 & 2.5 & 5.997 \\
\hline 4 & 1.058 & 1.455 & 1.522 & 2.144 & 1.007 & 0.905 & 1.786 & 0.752 & 1.403 & 1.984 \\
\hline 5 & 1.221 & 1.659 & 1.742 & 2.036 & 0.889 & 1.057 & 1.271 & 1.078 & 1.660 & 1.25 \\
\hline 6 & 0.379 & 0.805 & 0.483 & 0.812 & 1.009 & 0.861 & 1.238 & 0.800 & 0.767 & 0.95 \\
\hline 7 & 1.925 & 1.594 & 0.967 & 1.332 & 1.074 & 0.817 & 0.928 & 0.772 & 0.994 & 1.024 \\
\hline 8 & 0.804 & 1.005 & 0.999 & 0.912 & 1.069 & 0.782 & 0.831 & 0.997 & 0.879 & 0.943 \\
\hline 9 & 0.320 & 0.489 & 0.502 & 0.450 & 0.884 & 0.491 & 0.729 & 0.748 & 0.664 & 0.959 \\
\hline 10 & 1.181 & 1.162 & 1.028 & 0.980 & 1.218 & 1.165 & 0.951 & 1.079 & 0.621 & 0.791 \\
\hline
\end{tabular}

\section{Notes.}

${ }^{a}$ Table gives $\chi^{2}$ per degree of freedom of the independent spectrum estimates per multipole per band or band pair, estimated from the template-cleaned maps. See the text for details.

$\mathrm{b}$ The second header row indicates the number of degrees of freedom in the reduced $\chi^{2}$ for that spectrum. See the text for details.

spectrum for $\mathrm{KaQ}$. With 50 degrees of freedom, this is a $3 \sigma$ deviation, but given that we have $150 l \geqslant 6$ samples in the table, we expect of order 1 such value. Thus we conclude that the Fisher-based errors provide a good description of the DA-to-DA and year-to-year scatter in the $l \geqslant 6$ polarization data. If anything, there is a slight tendency to overestimate the uncertainties at higher $l$.

2 . For $l \leqslant 5$, we find 37 out of 120 points where the reduced $\chi^{2}$ deviates from 1 at more than $4 \sigma$ significance, indicating excessive internal scatter in the data relative to the Fisher errors. However, all but five of these occur in cross-power spectra in which one or both of the bands contain Wband data. If we exclude combinations with the $\mathrm{W}$ band, the remaining 72 points have a mode in the reduced $\chi^{2}$ distribution of 1 with a slight positive skewness due to the 5 points noted above, which all contain Q-band data. This may be a sign of slight foreground residuals contributing additional noise to the Q-band data, though we do not see similar evidence in the Ka-band spectra which would be more foreground contaminated prior to cleaning. For $\mathrm{Ka}-\mathrm{V}$ bands, we believe that the Fisher errors provide an adequate description of the scatter in this $l \leqslant 5$ polarization data, but we subject polarization sensitive cosmological parameter estimates, e.g., the optical depth, to additional scrutiny in Section 6.3.

3. Of special note is $l=3 \mathrm{BB}$ which, as noted in Page et al. (2007), is the power spectrum mode that is least modulated in the WMAP time-ordered data. This mode is therefore quite sensitive to how the instrument baseline is estimated and removed and, in turn, to how the $1 / \mathrm{f}$ noise is modeled. In the accounting above, the $l=3 \mathrm{BB}$ data have the highest internal scatter of any low-l polarization mode. In particular, every combination that includes W-band data is significantly discrepant; and the two most discrepant non-W-band points are also estimates of $l=3 \mathrm{BB}$. We comment on the W-band data further below, but note here that the final co-added BB spectrum (based on Ka-, Q-, and V-band data) does not lead to a significant detection of tensor modes. However, we caution that any surprising scientific conclusions that rely heavily on the WMAP $l=3$ $\mathrm{BB}$ data should be treated with caution.

Based on the analysis presented above, we find that the W-band polarization data are still too unstable at low- $l$ to be reliably used for cosmological studies. We cite more specific phenomenology and consider some possible explanations in the remainder of this section.

The five-year co-added W-band EE spectrum is shown in Figures 8 , in the form of likelihood profiles from $l=2-7$. At each multipole we show two curves: an estimate based on evaluating the likelihood multipole by multipole, and an estimate based on the pseudo- $C_{l}$ method (Page et al. 2007). The best-fit model EE spectrum, based on the combined Ka-, Q-, and V-band data is indicated by the dashed lines in each panel. Both spectrum estimates show excess power relative to the model spectrum, with the most puzzling multipole being $l=7$ which, as shown in Table 5, has an internal reduced $\chi^{2}$ of 1.015 , for 190 degrees of freedom. These data have the hallmark of a sky signal, but that hypothesis is implausible for a variety of reasons (Page et al. 2007). It is more likely due to a systematic effect that is common to a majority of the W-band channels over a majority of the five years of data. We explore and rule out one previously neglected effect in Section 6.2. It is worth recalling that $l=7 \mathrm{EE}$, like $l=3 \mathrm{BB}$, is a mode that is relatively poorly measured by WMAP, as discussed in Page et al. (2007, see especially Figure 16 and its related discussion).

The W-band BB data also exhibit unusual behavior at $l=2,3$. In this case, these two multipoles have internal reduced $\chi^{2}$ greater than 6 , and the co-added $l=2$ point is nearly $10 \sigma$ from zero. However, with 190 points in each fiveyear co-added estimate it is now possible to look for trends within the data that were relatively obscure with only three years of data. In particular, we note that in the $l=2$ estimate, there are 28 points that are individually more than $5 \sigma$ from zero and that all of them contain $\mathrm{W} 1$ data in one or both of the DA pairs in the cross-power spectrum. Similarly for $l=3$, there are 14 points greater than $5 \sigma$ and all of those points contain W4 data in one or both of the DA pairs. We have yet to pinpoint the significance of this result, but we plan to study the noise properties of these DAs beyond what has been reported to date, and to sharpen the phenomenology with additional years of data.

\subsection{Emissivity Tests}

In this section we consider time-dependent emission from the $W M A P$ optics as a candidate for explaining the excess W-band 

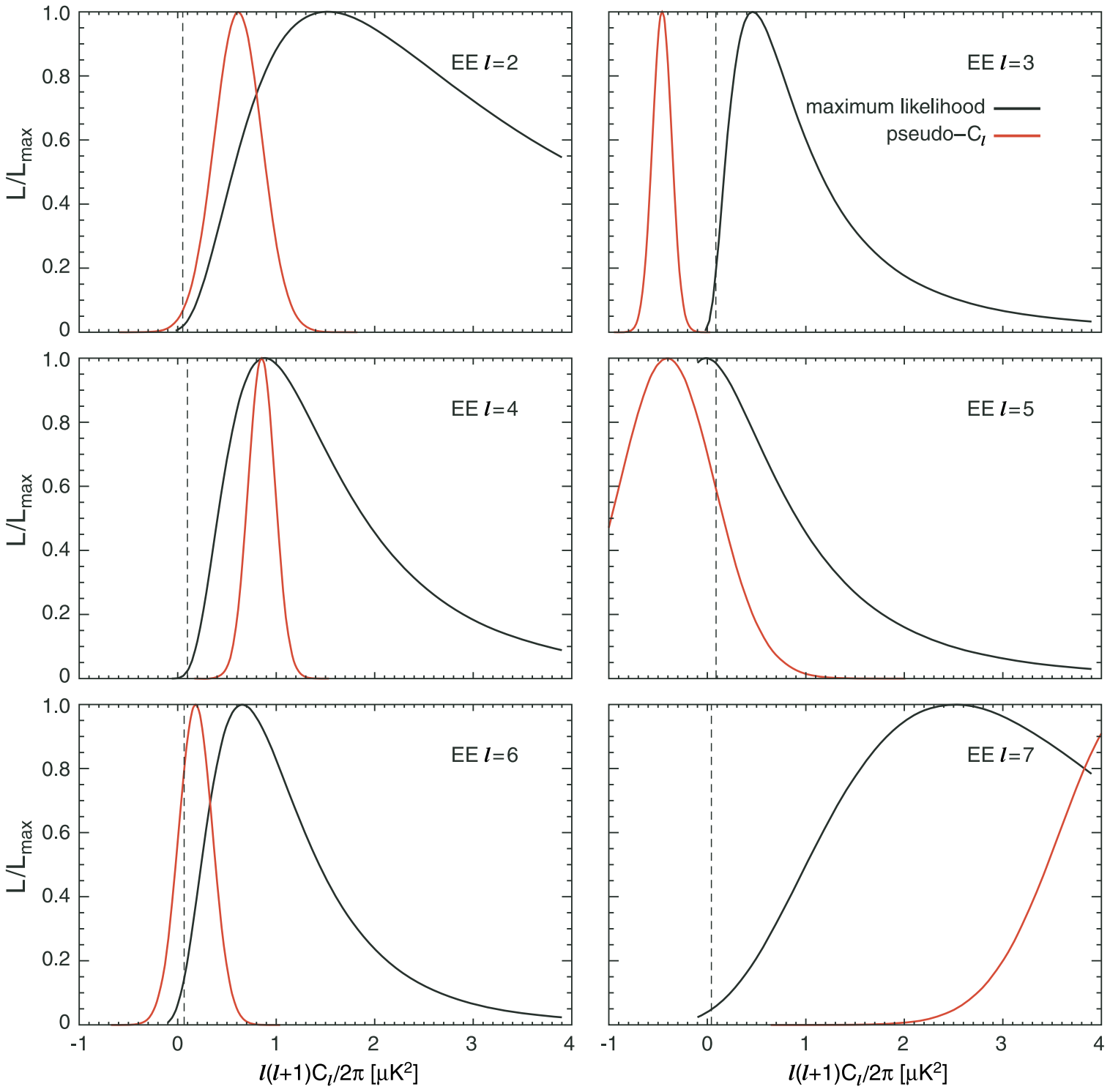

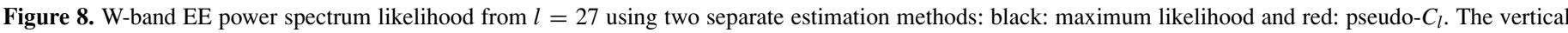

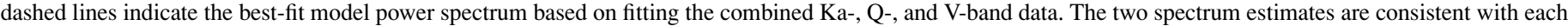

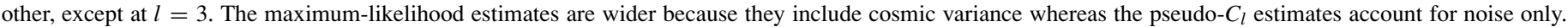

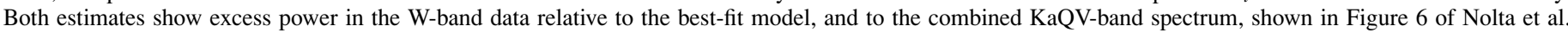

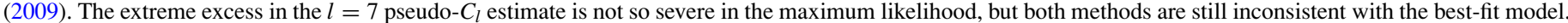

"signal" seen in the EE spectrum, mostly at $l=7$. In the end, the effect proved not to be significant, but it provides a useful illustration of a common-mode effect that we believe is still present in the $\mathrm{W}$-band polarization data.

From a number of lines of reasoning, we know that the microwave emissivity of the mirrors is a few percent in the $\mathrm{W}$ band, and that it scales with frequency roughly like $v^{1.5}$ across the WMAP frequency range, as expected for a classical metal (Born \& Wolf 1980). Hence this mechanism has the potential to explain a common-mode effect that is primarily seen in the W band. Further, Figure 1 in Jarosik et al. (2007) shows that the physical temperature of the primary mirrors are modulated at the spin period by $\sim 200 \mu \mathrm{K}$, with a dependence on the solar azimuth angle that is highly repeatable from year to year. We believe that this modulation is driven by solar radiation diffracting around the WMAP Sun shield reaching the tops of the primary mirrors, which are only a few degrees within the geometric shadow of the Sun shield. In contrast, the secondary mirrors and feed horns are in deep shadow and show no measurable variation at the spin period, so that any emission they produce only contributes to an overall radiometer offset, and will not be further considered here.

As a rough estimate, the spin-modulated emission from the primary mirrors could produce as much as $\sim 0.02 \times 200=$ $4 \mu \mathrm{K}$ of radiometric response in the $\mathrm{W}$ band, but the actual signal depends on the relative phase of the A- and B-side mirror variations and the polarization state of the emission. In more detail, the differential signal, $d(t)$, measured by a radiometer with lossy elements is

$$
d(t)=\left(1-\epsilon_{A}\right) T_{A}(t)-\left(1-\epsilon_{B}\right) T_{B}(t)+\epsilon_{A}^{\mathrm{p}} T_{A}^{\mathrm{p}}(t)-\epsilon_{B}^{\mathrm{p}} T_{B}^{\mathrm{p}}(t),
$$

where $\epsilon_{A}=\epsilon_{A}^{\mathrm{p}}+\epsilon_{A}^{\mathrm{s}}+\epsilon_{A}^{\mathrm{f}}$ is the combined loss in the Aside optics: primary (p) plus secondary (s) mirrors, plus the feed (f) horn, and likewise for the B-side. $T_{A, B}$ is the sky temperature in the direction of the A- or B-side line-of-sight; 
Table 6

Loss Imbalance Coefficients ${ }^{\mathrm{a}}$

\begin{tabular}{lrc}
\hline \hline DA & $\begin{array}{c}x_{\mathrm{im}, 1} \\
(\%)\end{array}$ & $\begin{array}{c}x_{\mathrm{im}, 2} \\
(\%)\end{array}$ \\
\hline K1 & 0.012 & 0.589 \\
Ka1 & 0.359 & 0.148 \\
Q1 & -0.031 & 0.412 \\
Q2 & 0.691 & 1.048 \\
V1 & 0.041 & 0.226 \\
V2 & 0.404 & 0.409 \\
W1 & 0.939 & 0.128 \\
W2 & 0.601 & 1.140 \\
W3 & 0.009 & 0.497 \\
W4 & 2.615 & 1.946 \\
\hline
\end{tabular}

Notes.

a Loss imbalance is defined as $x_{\mathrm{im}}=\left(\epsilon_{A}-\epsilon_{B}\right) /\left(\epsilon_{A}+\epsilon_{B}\right)$. See Section 6.2 and Jarosik et al. (2007) for details.

and $T_{A, B}^{\mathrm{p}}$ is the physical temperature of the A- or B-side primary mirror.

The first two terms are the sky signal attenuated by the overall loss in the A- and B- side optics, respectively. The effects of loss imbalance, which arise when $\epsilon_{A} \neq \epsilon_{B}$, have been studied extensively (Jarosik et al. 2003, 2007). We account for loss imbalance in the data processing and we marginalize over residual uncertainties in the imbalance coefficients when we form the pixel-pixel inverse covariance matrices (Jarosik et al. 2007). Updated estimates of the loss imbalance coefficients based on fits to the five-year data are reported in Table 6.

In the remainder of this section we focus on the last two emissive terms in Equation (10). Recall that a WMAP DA consists of two radiometers, 1 and 2 , that are sensitive to orthogonal linear polarization modes. The temperature and polarization signals are extracted by forming the sum and difference of the two radiometer outputs; thus, the emission terms we need to evaluate are

$$
d_{1}^{\mathrm{p}}(t) \pm d_{2}^{\mathrm{p}}(t)=\left(\frac{\epsilon_{A 1}^{\mathrm{p}} \pm \epsilon_{A 2}^{\mathrm{p}}}{1-\epsilon}\right) T_{A}^{\mathrm{p}}-\left(\frac{\epsilon_{B 1}^{\mathrm{p}} \pm \epsilon_{B 2}^{\mathrm{p}}}{1-\epsilon}\right) T_{B}^{\mathrm{p}},
$$

where $\epsilon_{A 1}^{\mathrm{p}}$ is the A-side primary mirror emissivity measured by radiometer 1 , and so forth. The factor of $1-\epsilon$ in the denominator applies a small correction for the mean loss, $\epsilon \equiv\left(\epsilon_{A}+\epsilon_{B}\right) / 2$, and arises from the process of calibrating the data to a known sky brightness temperature (Section 4$)$. Note that we only pick up a polarized response if $\epsilon_{1} \neq \epsilon_{2}$.

We have simulated this signal in the time-ordered data using the measured primary mirror temperatures as template inputs. The emissivity coefficients were initially chosen to be consistent with the loss imbalance constraints. However, in order to produce a measurable polarization signal, we had to boost the emissivity differences to the point where they became unphysical, that is $\left|\epsilon_{1}-\epsilon_{2}\right|>\left|\epsilon_{1}+\epsilon_{2}\right|$. Nonetheless, it was instructive to analyze this simulation by binning the resulting data (which also includes sky signal and noise) as a function of solar azimuth. The results are shown in the top panel of Figure 9 which shows three years of co-added W-band polarization data, the $d_{1}-d_{2}$ channel; the input emissive signal is shown in red for comparison. We are clearly able to detect such a signal with this manner of binning. We also computed the low- $l$ polarization spectra and found that, despite the large spin-modulated input signal, the signal induced in the power spectrum was less than $2 \mu \mathrm{K}^{2}$ in $l(l+1) C_{l}^{E E} / 2 \pi$, which is insufficient to explain the $l=7$ feature in the W-band EE spectrum.
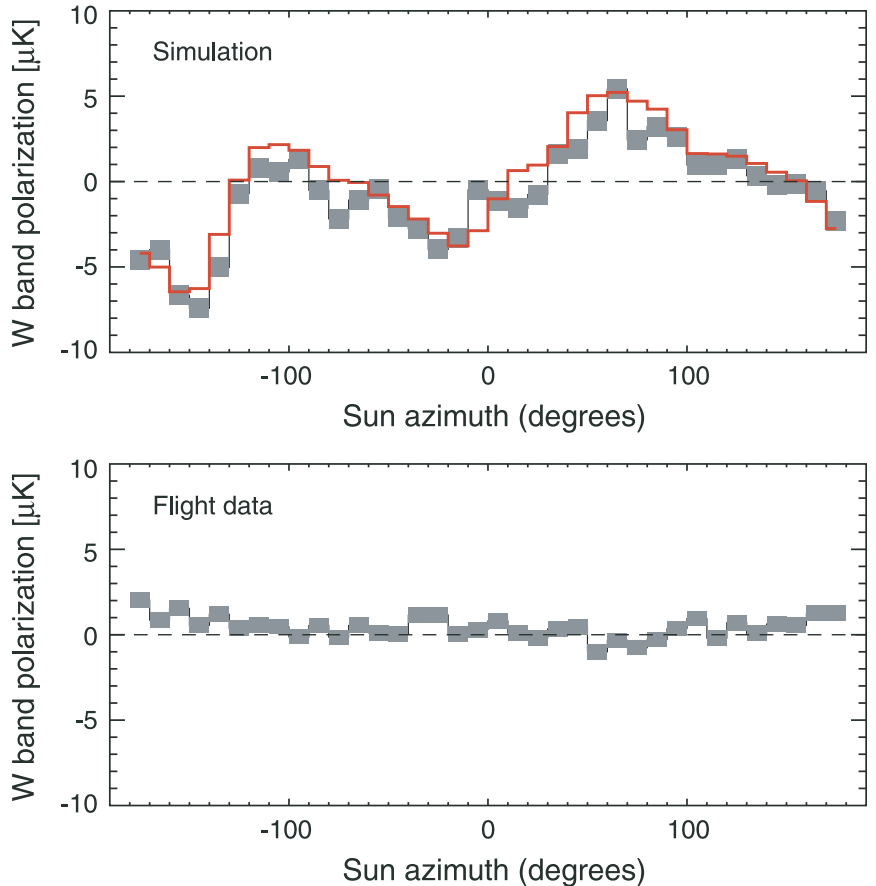

Figure 9. Top: simulated W-band data with a large polarized thermal emission signal injected, binned by the solar azimuth angle. The red trace shows the input waveform based on the flight mirror temperature profile and a model of the polarized emissivity. The black profile is the binned co-added data which follow the input signal very well. The thickness of the points represents the $1 \sigma$ uncertainty due to white noise. bottom: same as the top panel but for the five year flight data. The reduced $\chi^{2}$ of the binned data with respect to zero is 2.1 for 36 degrees of freedom, but this does not account for $1 / f$ noise, so the significance of this result requires further investigation. However, the much larger signal in the simulation did not produce an EE spectrum with features present in the flight W-band EE spectrum, so the feature in the binned flight data cannot account for the excess $l=7$ emission.

In parallel with the simulation analysis, we have binned the flight radiometer data by the solar azimuth angle to search for spin-modulated features in the polarization data. The results for the $\mathrm{W}$ band are shown in the bottom panel of Figure 9 for the five-year data. While the $\chi^{2}$ per degree of freedom relative to zero is slightly high, there is no compelling evidence for a coherent spin-modulated signal at the $\sim 2 \mu \mathrm{K}$ level. In contrast, the simulation yielded spin-modulated signals of $5-10 \mu \mathrm{K}$ and still failed to produce a significant effect in the EE spectrum. Hence we conclude that thermal emission from the WMAP optics cannot explain the excess W-band EE signal. In any event, we continue to monitor the spin-modulated data for the emergence of a coherent signal.

\subsection{Ka-Band Tests}

The analysis presented in Section 6.1 shows that the Ka-band polarization data are comparable to the Q- and V-band data in its internal consistency. That analysis was performed on data that had been foreground cleaned using the template method discussed in Page et al. (2007) and updated in Gold et al. (2009). In order to assess whether or not these cleaned Ka-band data are suitable for use in cosmological parameter estimation we subject it to two further tests: (1) a null test in which Ka-band data are compared to the combined Q- and V-band data, and (2) a parameter estimation based solely on Ka-band data.

For the null test, we form polarization maps by taking differences, $\frac{1}{2} S_{\mathrm{Ka}}-\frac{1}{2} S_{\mathrm{QV}}$, where $S=Q, U$ are the polarization Stokes parameters, $S_{\mathrm{Ka}}$ are the maps formed from the Ka-band 


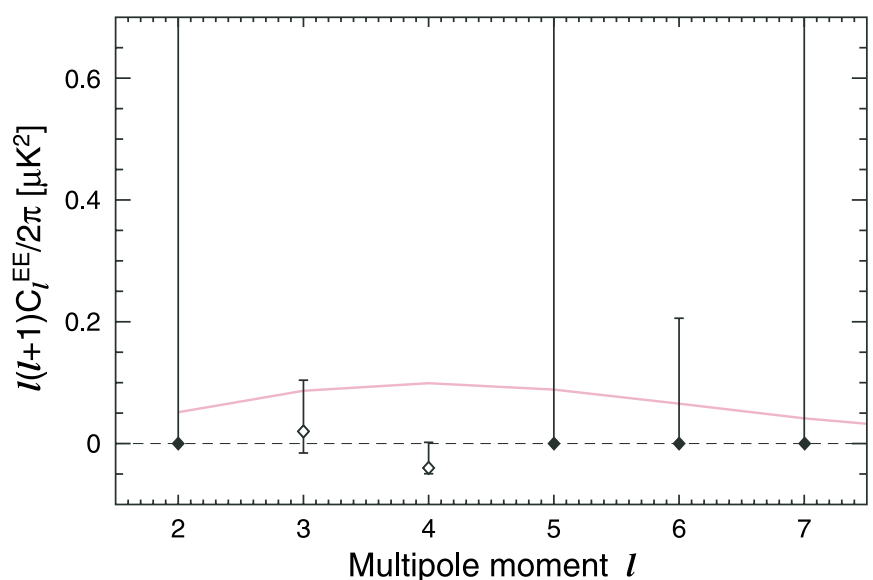

Figure 10. The EE power spectrum computed from the null sky maps, $\frac{1}{2} S_{\mathrm{Ka}}-\frac{1}{2} S_{\mathrm{QV}}$, where $S=Q, U$ are the polarization Stokes parameters, and $S_{\mathrm{QV}}$ is the optimal combination of the Q- and V-band data. The pink curve is the best-fit theoretical spectrum from Dunkley et al. (2009). The spectrum derived from the null maps is consistent with zero.

data, and $S_{\mathrm{QV}}$ are the maps formed from the optimal combination of the Q- and V-band data. We evaluate the EE power spectrum from these null maps by evaluating the likelihood mode by mode while holding the other multipoles fixed at zero. The results are shown in Figure 10, along with the best-fit model spectrum based on the final five-year $\Lambda \mathrm{CDM}$ analysis. The spectrum is clearly consistent with zero, but to get a better sense of the power of this test, we have also used these null maps to estimate the optical depth parameter, $\tau$. The result of that analysis is shown as the dashed curve in Figure 11, where we find that the null likelihood peaks at $\tau=0$ and excludes the most-likely cosmological value with $\sim 95 \%$ confidence.

As a separate test, we evaluate the $\tau$ likelihood using only the template-cleaned Ka-band signal maps. The result of that test is shown as the blue curve in Figure 11. While the uncertainty in the Ka-band estimate is considerably larger than the combined QV estimate (shown in red), the estimates are highly consistent. The result of combining Ka-, Q-, and V-band data is shown in the black curve.

Dunkley et al. (2009) present a complementary method of foreground cleaning that makes use of Ka-band data, in conjunction with K-, Q-, and V-band data. Using a full sixparameter likelihood evaluation, they compare the optical depth inferred from the two cleaning methods while using the full combined data sets in both cases: see Figure 9 of Dunkley et al. (2009) for details. Based on these tests, we conclude that the Ka-band data are sufficiently free of systematic errors and residual foreground signals that it is suitable for cosmological studies. The use of this band significantly enhances the overall polarization sensitivity of the WMAP data.

\section{SUMMARY OF FIVE-YEAR SCIENCE RESULTS}

Detailed presentations of the scientific results from the fiveyear data are given by Gold et al. (2009), Wright et al. (2009), Nolta et al. (2009), Dunkley et al. (2009), and Komatsu et al. (2009). Starting with the five-year temperature and polarization maps, with their improved calibration, Gold et al. (2009) give a new Markov Chain Monte Carlo-based analysis of foreground emission in the data. Their results are broadly consistent with previous analyses by the WMAP team and others (Eriksen et al. $2007 \mathrm{~b}$ ), while providing some new results on the microwave spectra of bright sources in the Galactic plane that are not well

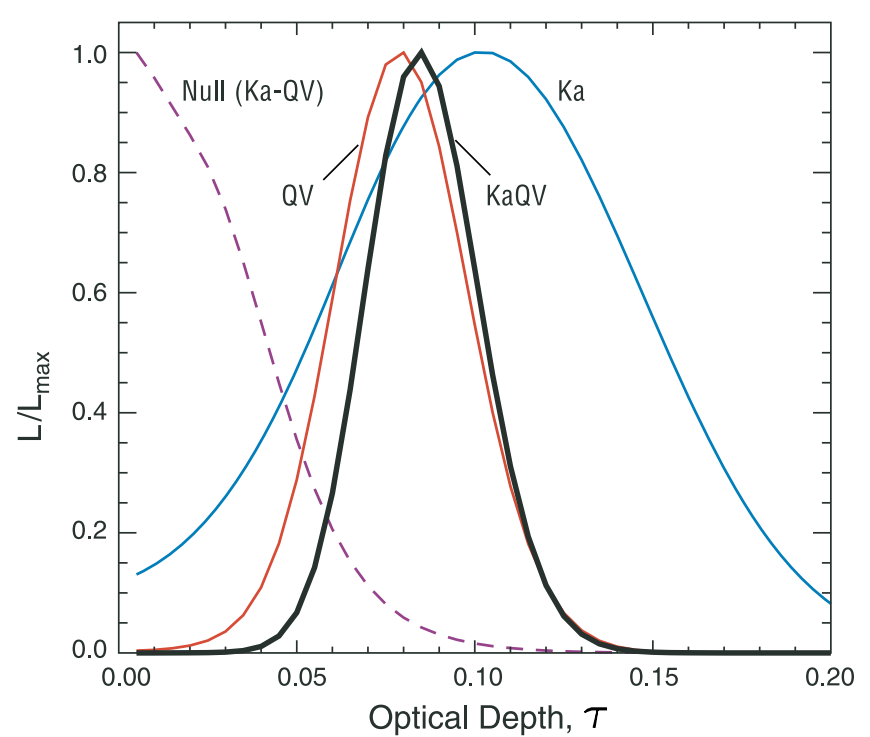

Figure 11. Estimates of the optical depth from a variety of data combinations. The dashed curve labeled Null uses the same null sky maps used in Figure 10. The optical depth obtained from Ka-band data alone (blue) is consistent with independent estimates from the combined Q- and V-band data (red). The final five year analysis uses Ka-, Q-, and V-band data combined (black). These estimates all use a one-parameter likelihood estimation, holding other parameters fixed except for the fluctuation amplitude, which is adjusted to fit the first acoustic peak in the TT spectrum (Page et al. 2007). The degeneracy between $\tau$ and other $\Lambda$ CDM parameters is small: see Figure 7 of Dunkley et al. (2009).

fitted by simple power-law foreground models. Figure 12 shows the five-year CMB map based on the internal linear combination (ILC) method of foreground removal.

Wright et al. (2009) give a comprehensive analysis of the extragalactic sources in the five-year data, including a new analysis of variability made possible by the multiyear coverage. The five-year WMAP source catalog now contains 390 objects and is reasonably complete to a flux of $1 \mathrm{Jy}$ away from the Galactic plane. The new analysis of the WMAP beam response (Hill et al. 2009) has led to more precise estimates of the point source flux scale for all five WMAP frequency bands. This information is incorporated in the new source catalog (Wright et al. 2009), and is also used to provide new brightness estimates of Mars, Jupiter, and Saturn (Hill et al. 2009). We find significant (and expected) variability in Mars and Saturn over the course of five years and use that information to provide a preliminary recalibration of a Mars brightness model (Wright 2007), and to fit a simple model of Saturn's brightness as a function of ring inclination.

The temperature and polarization power spectra are presented in Nolta et al. (2009). The spectra are all consistent with the three-year results with improvements in sensitivity commensurate with the additional integration time. Further improvements in our understanding of the absolute calibration and beam response have allowed us to place tighter uncertainties on the power spectra, over and above the reductions from additional data. These changes are all reflected in the new version of the WMAP likelihood code. The most notable improvements arise in the third acoustic peak of the TT spectrum, and in all of the polarization spectra; for example, we now see unambiguous evidence for a second dip in the high- $l$ TE spectrum, which further constrains deviations from the standard $\Lambda$ CDM model. The five-year TT and TE spectra are shown in Figure 13. We have also generated new maximum-likelihood estimates of the low- $l$ polarization spectra: TE, TB, EE, EB, and BB to complement 


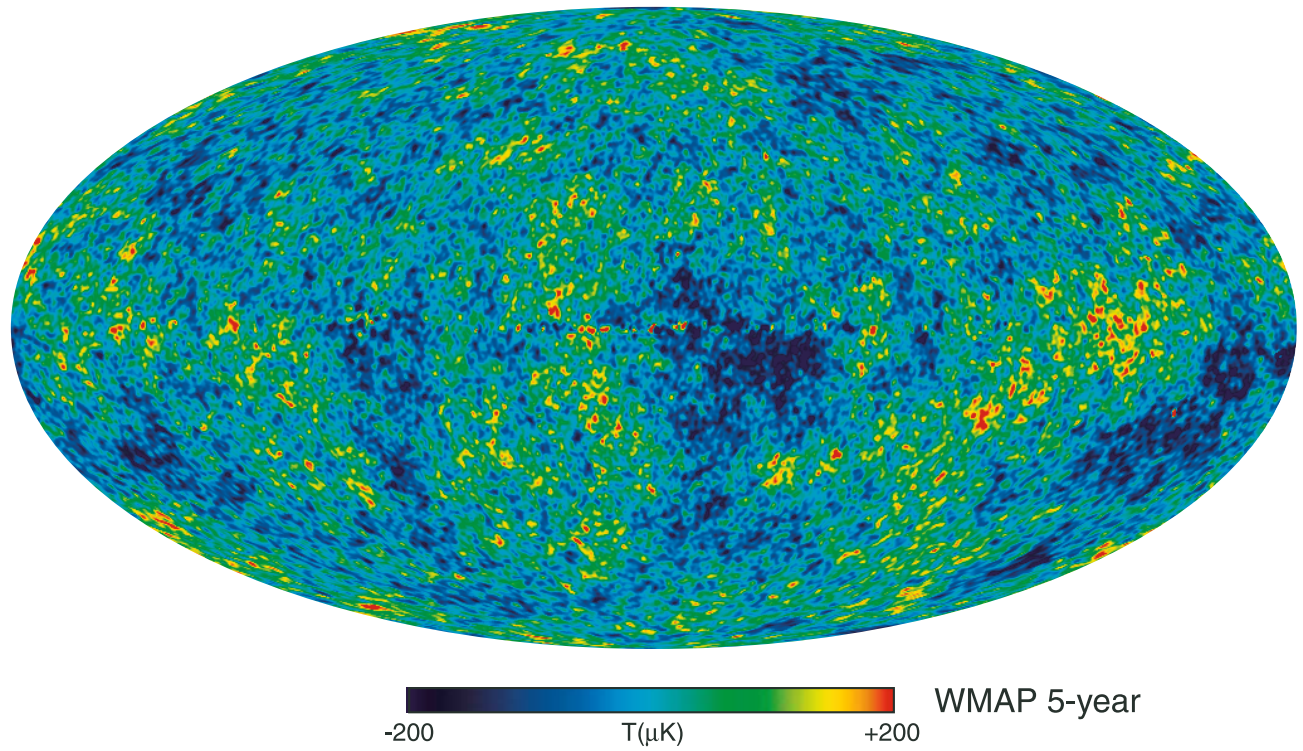

Figure 12. The foreground-reduced Internal Linear Combination (ILC) map based on the five year WMAP data.

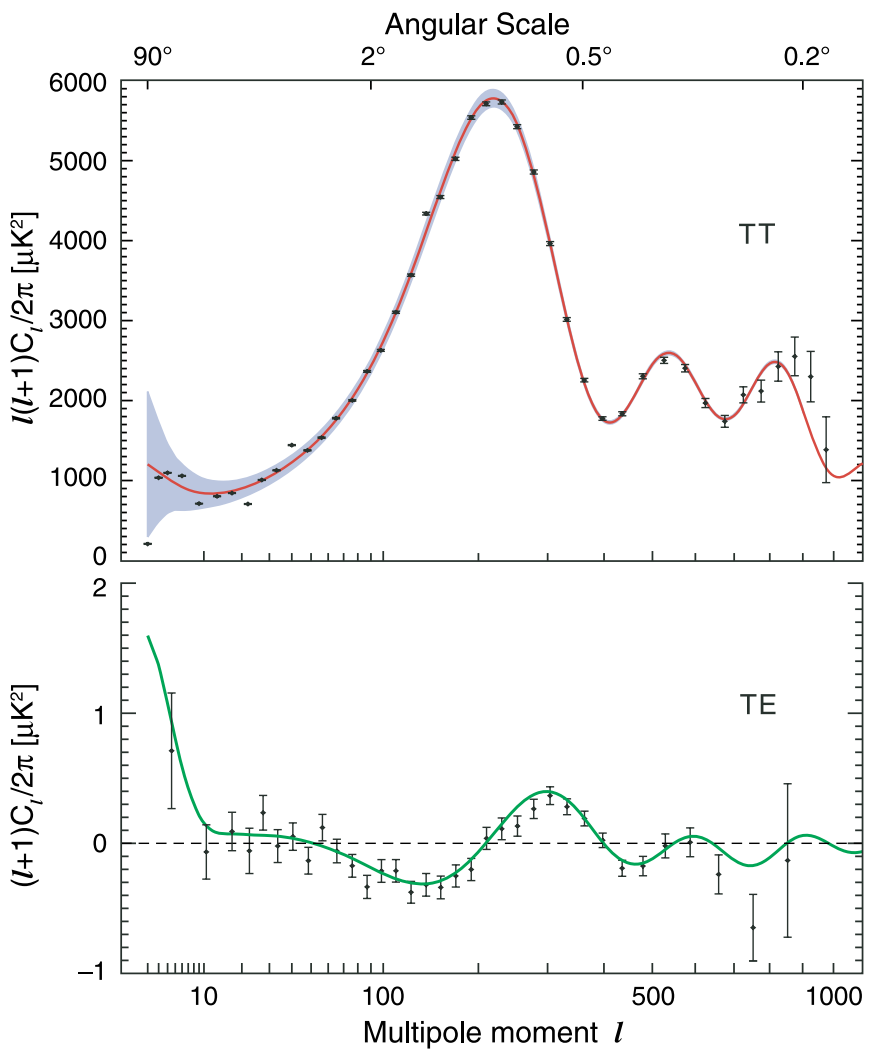

Figure 13. The temperature (TT) and temperature-polarization correlation (TE) power spectra based on the five year WMAP data. The addition of two years of data provides more sensitive measurements of the third peak in the TT and the high- $l$ TE spectra, especially the second trough.

our earlier estimates based on pseudo- $C_{l}$ methods (Nolta et al. 2009). The TB, EB, and BB spectra remain consistent with zero.

The cosmological implications of the five-year WMAP data are discussed in detail in Dunkley et al. (2009) and Komatsu et al. (2009). The now-standard cosmological model: a flat universe dominated by vacuum energy and dark matter, seeded by nearly scale-invariant, adiabatic, Gaussian random-phase fluctuations, continues to fit the five-year data. WMAP has now determined the key parameters of this model to high precision; a summary of the five-year parameter results is given in Table 7. The most notable improvements are the measurements of the dark matter density, $\Omega_{c} h^{2}$, and the amplitude of matter fluctuations today, $\sigma_{8}$. The former is determined with $6 \%$ uncertainty using $W M A P$ data only (Dunkley et al. 2009), and with 3\% uncertainty when WMAP data are combined with $\mathrm{BAO}$ and $\mathrm{SNe}$ constraints (Komatsu et al. 2009). The latter is measured to 5\% with WMAP data, and to $3 \%$ when combined with other data. The redshift of reionization is $z_{\text {reion }}=11.0 \pm 1.4$, if the universe were reionized instantaneously. The $2 \sigma$ lower limit is $z_{\text {reion }}>8.2$, and instantaneous reionization at $z_{\text {reion }}=6$ is rejected at $3.5 \sigma$. The WMAP data continue to favor models with a tilted primordial spectrum, $n_{s}=0.963_{-0.015}^{+0.014}$. Dunkley et al. (2009) discuss how the $\Lambda \mathrm{CDM}$ model continues to fit a host of other astronomical data as well.

Moving beyond the standard $\Lambda \mathrm{CDM}$ model, when WMAP data are combined with $\mathrm{BAO}$ and $\mathrm{SNe}$ observations (Komatsu et al. 2009), we find no evidence for running in the spectral index of scalar fluctuations, $d n_{s} / d \ln k=-0.028 \pm 0.020$ $(68 \% \mathrm{CL})$. The new limit on the tensor-to-scalar ratio is $r<0.22$ (95\% CL), and we obtain tight, simultaneous limits on the (constant) dark energy equation of state and the spatial curvature of the universe: $-0.14<1+w<0.12$ (95\% CL) and $-0.0179<\Omega_{k}<0.0081(95 \% \mathrm{CL})$. The angular power spectrum now exhibits the signature of the cosmic neutrino background: the number of relativistic degrees of freedom, expressed in units of the effective number of neutrino species, is found to be $N_{\text {eff }}=4.4 \pm 1.5(68 \%$ CL), consistent with the standard value of 3.04. Models with $N_{\text {eff }}=0$ are disfavored at $>99.5 \%$ confidence. A summary of the key cosmological parameter values is given in Table 7, where we provide estimates using WMAP data alone and WMAP data combined with BAO and $\mathrm{SNe}$ observations. A complete tabulation of all parameter values for each model and data set combination we studied is available on LAMBDA.

The new data also place more stringent limits on deviations from Gaussianity, parity violations, and the amplitude of isocurvature fluctuations (Komatsu et al. 2009). For example, new limits on physically motivated primordial non-Gaussianity 
Table 7

Cosmological Parameter Summary

\begin{tabular}{|c|c|c|c|}
\hline Description & Symbol & WMAP Only & $W M A P+\mathrm{BAO}+\mathrm{SN}$ \\
\hline \multicolumn{4}{|c|}{ Parameters for Standard $\Lambda$ CDM Model ${ }^{a}$} \\
\hline Age of universe & $t_{0}$ & $13.69 \pm 0.13 \mathrm{Gyr}$ & $13.72 \pm 0.12 \mathrm{Gyr}$ \\
\hline Hubble constant & $H_{0}$ & $71.9_{-2.7}^{+2.6} \mathrm{~km} \mathrm{~s}^{-1} \mathrm{Mpc}^{-1}$ & $70.5 \pm 1.3 \mathrm{~km} \mathrm{~s}^{-1} \mathrm{Mpc}^{-1}$ \\
\hline Baryon density & $\Omega_{b}$ & $0.0441 \pm 0.0030$ & $0.0456 \pm 0.0015$ \\
\hline Physical baryon density & $\Omega_{b} h^{2}$ & $0.02273 \pm 0.00062$ & $0.02267_{-0.00059}^{+0.00058}$ \\
\hline Dark matter density & $\Omega_{c}$ & $0.214 \pm 0.027$ & $0.228 \pm 0.013$ \\
\hline Physical dark matter density & $\Omega_{c} h^{2}$ & $0.1099 \pm 0.0062$ & $0.1131 \pm 0.0034$ \\
\hline Dark energy density & $\Omega_{\Lambda}$ & $0.742 \pm 0.030$ & $0.726 \pm 0.015$ \\
\hline Curvature fluctuation amplitude, $k_{0}=0.002 \mathrm{Mpc}^{-1 \mathrm{~b}}$ & $\Delta_{\mathcal{R}}^{2}$ & $(2.41 \pm 0.11) \times 10^{-9}$ & $(2.445 \pm 0.096) \times 10^{-9}$ \\
\hline Fluctuation amplitude at $8 h^{-1} \mathrm{Mpc}$ & $\sigma_{8}$ & $0.796 \pm 0.036$ & $0.812 \pm 0.026$ \\
\hline$l(l+1) C_{220}^{T T} / 2 \pi$ & $C_{220}$ & $5756 \pm 42 \mu \mathrm{K}^{2}$ & $5751_{-43}^{+42} \mu \mathrm{K}^{2}$ \\
\hline Scalar spectral index & $n_{s}$ & $0.963_{-0.015}^{+0.014}$ & $0.960 \pm 0.013$ \\
\hline Redshift of matter-radiation equality & $z_{\text {eq }}$ & $3176_{-150}^{+151}$ & $3253_{-87}^{+89}$ \\
\hline Angular diameter distance to matter-radiation eq. ${ }^{\mathrm{c}}$ & $d_{A}\left(z_{\mathrm{eq}}\right)$ & $14279_{-189}^{+186} \mathrm{Mpc}$ & $14200_{-140}^{+13 \overline{7}^{\circ}} \mathrm{Mpc}$ \\
\hline Redshift of decoupling & $z_{*}$ & $1090.51 \pm 0.95$ & $1090.88 \pm 0.72$ \\
\hline Age at decoupling & $t_{*}$ & $380081_{-5841}^{+5843} \mathrm{yr}$ & $376971_{-3167}^{+3162} \mathrm{yr}$ \\
\hline Angular diameter distance to decoupling ${ }^{\mathrm{c}, \mathrm{d}}$ & $d_{A}\left(z_{*}\right)$ & $14115_{-191}^{+188} \mathrm{Mpc}$ & $14034_{-142}^{+138} \mathrm{Mpc}$ \\
\hline Sound horizon at decoupling ${ }^{d}$ & $r_{S}\left(z_{*}\right)$ & $146.8 \pm 1.8 \mathrm{Mpc}$ & $145.9_{-1.2}^{+1.1} \mathrm{Mpc}$ \\
\hline Acoustic scale at decoupling ${ }^{d}$ & $l_{A}\left(z_{*}\right)$ & $302.08_{-0.84}^{+0.83}$ & $302.13 \pm 0.84$ \\
\hline Reionization optical depth & $\tau$ & $0.087 \pm 0.017$ & $0.084 \pm 0.016$ \\
\hline Redshift of reionization & $z_{\text {reion }}$ & $11.0 \pm 1.4$ & $10.9 \pm 1.4$ \\
\hline Age at reionization & $t_{\text {reion }}$ & $427_{-65}^{+88} \mathrm{Myr}$ & $432_{-67}^{+90} \mathrm{Myr}$ \\
\hline \multicolumn{4}{|c|}{ Parameters for Extended Models $\mathrm{e}$} \\
\hline Total density $\mathrm{f}$ & $\Omega_{\text {tot }}$ & $1.099_{-0.085}^{+0.100}$ & $1.0050_{-0.0061}^{+0.0060}$ \\
\hline Equation of state ${ }^{g}$ & $w$ & $-1.06_{-0.42}^{+0.41}$ & $-0.992_{-0.062}^{+0.0601}$ \\
\hline Tensor to scalar ratio, $k_{0}=0.002 \mathrm{Mpc}^{-1 \mathrm{~b}, \mathrm{~h}}$ & $r$ & $<0.43(95 \% \mathrm{CL})$ & $<0.22(95 \% \mathrm{CL})$ \\
\hline Running of spectral index, $k_{0}=0.002 \mathrm{Mpc}^{-1 \mathrm{~b}, \mathrm{i}}$ & $d n_{s} / d \ln k$ & $-0.037 \pm 0.028$ & $-0.028 \pm 0.020$ \\
\hline Neutrino density $\mathrm{j}$ & $\Omega_{v} h^{2}$ & $<0.014(95 \% \mathrm{CL})$ & $<0.0071(95 \% \mathrm{CL})$ \\
\hline Neutrino mass $\mathrm{j}$ & $\sum m_{v}$ & $<1.3 \mathrm{eV}(95 \% \mathrm{CL})$ & $<0.67 \mathrm{eV}(95 \% \mathrm{CL})$ \\
\hline Number of light neutrino families $\mathrm{k}$ & $N_{\text {eff }}$ & $>2.3(95 \% \mathrm{CL})$ & $4.4 \pm 1.5$ \\
\hline
\end{tabular}

Notes.

a The parameters reported in the first section assume the six-parameter $\Lambda$ CDM model, first using WMAP data only (Dunkley et al. 2009), and then using WMAP+BAO+SN data (Komatsu et al. 2009).

${ }^{\mathrm{b}} k=0.002 \mathrm{Mpc}^{-1} \longleftrightarrow l_{\text {eff }} \approx 30$.

c Comoving angular diameter distance.

$\mathrm{d} l_{A}\left(z_{*}\right) \equiv \pi d_{A}\left(z_{*}\right) r_{s}\left(z_{*}\right)^{-1}$.

e The parameters reported in the second section place limits on deviations from the $\Lambda$ CDM model, first using $W M A P$ data only (Dunkley et al. 2009), and then using WMAP+BAO+SN data (Komatsu et al. 2009). A complete listing of all parameter values and uncertainties for each of the extended models studied is available on LAMBDA.

${ }^{\mathrm{f}}$ Allows nonzero curvature, $\Omega_{k} \neq 0$.

g Allows $w \neq-1$, but assumes $w$ is constant.

${ }^{\mathrm{h}}$ Allows tensors modes but no running in the scalar spectral index.

${ }^{\mathrm{i}}$ Allows running in the scalar spectral index but no tensor modes.

j Allows a massive neutrino component, $\Omega_{v} \neq 0$.

${ }^{\mathrm{k}}$ Allows $N_{\mathrm{eff}}$ number of relativistic species. The last column adds the Hubble Space Telescope (HST) prior to the other data sets.

parameters are $-9<f_{\mathrm{NL}}^{\text {local }}<111(95 \% \mathrm{CL})$ and $-151<$ $f_{\mathrm{NL}}^{\text {equil }}<253(95 \% \mathrm{CL})$ for the local and equilateral models, respectively.

\section{CONCLUSIONS}

We have presented an overview of the five-year WMAP data and have highlighted the improvements we have made to the data processing and analysis since the three-year results were presented. The most substantive improvements to the processing include a new method for establishing the absolute gain calibration (with reduced uncertainty), and a more complete analysis of the WMAP beam response made possible by additional data and a higher fidelity physical optics model. Numerous other processing changes are outlined in Section 2.

The five-year sky maps are consistent with the three-year maps and have noise levels that are $\sqrt{5}$ times less than the single- year maps. The new maps are compared to the three-year maps in Section 3 . The main changes to the angular power spectrum are as follows: at low multipoles $(l \lesssim 100)$ the spectrum is $\sim 0.5 \%$ higher than the three-year spectrum (in power units) due to the new absolute gain determination. At higher multipoles it is increased by $\sim 2.5 \%$, due to the new beam response profiles, as explained in Section 5 and in Hill et al. (2009). These changes are consistent with the three-year uncertainties when one accounts for both the $0.5 \%$ gain uncertainty (in temperature units) and the three-year beam uncertainties, which were incorporated into the likelihood code.

We have applied a number of new tests to the polarization data to check internal consistency and to look for new systematic effects in the W-band data (Section 6). As a result of these tests, and of new analyses of polarized foreground emission (Dunkley et al. 2009), we have concluded that Ka-band data can be used along with Q- and V-band data for cosmological 
analyses. However, we still find a number of features in the $\mathrm{W}$-band polarization data that preclude its use, except in the Galactic plane where the signal to noise is relatively high. We continue to investigate the causes of this and have identified new clues to follow up on in future studies (Section 6.1).

Scientific results gathered from the suite of five-year papers are summarized in Section 7. The highlights include smaller uncertainties in the optical depth, $\tau$, due to a combination of additional years of data and to the inclusion of Ka-band polarization data: instantaneous reionization at $z_{\text {reion }}=6$ is now rejected at $3.5 \sigma$. New evidence favoring a non-zero neutrino abundance at the epoch of last scattering, made possible by improved measurements of the third acoustic peak; and new limits on the non-Gaussian parameter $f_{\mathrm{NL}}$, based on additional data and the application of a new, more optimal bispectrum estimator. The five-year data continue to favor a tilted primordial fluctuation spectrum, in the range $n_{s} \sim 0.96$, but a purely scale invariant spectrum cannot be ruled out at $>3 \sigma$ confidence.

The WMAP observatory continues to operate at L2 as designed, and the addition of two years of flight data has allowed us to make significant advances in characterizing the instrument. Additional data beyond five years will give us a better understanding of the instrument, especially with regards to the W-band polarization data since the number of jackknife combinations scales like the square of the number of years of operation. If W-band data can be incorporated into the EE power spectrum estimate, it would become possible to constrain a second reionization parameter and thereby further probe this important epoch in cosmology. The WMAP data continue to uphold the standard $\Lambda \mathrm{CDM}$ model but more data may reveal new surprises.

\section{DATA PRODUCTS}

All of the WMAP data is released to the research community for further analysis through the Legacy Archive for Microwave Background Data Analysis (LAMBDA) at http://lambda.gsfc.nasa.gov. The products include the complete five-year time-ordered data archive (both raw and calibrated); the calibrated sky maps in a variety of processing stages (single year by DA, multiyear by band, high resolution and low resolution, smoothed, foreground-subtracted, and so forth); the angular power spectra and cosmological model likelihood code; a full table of model parameter values for a variety of model and data sets (including the best-fit model spectra and Markov chains); and a host of ancillary data to support further analysis. The WMAP Explanatory Supplement provides detailed information about the WMAP in-flight operations and data products (Limon et al. 2008).

The WMAP mission is made possible by the support of the Science Mission Directorate Office at NASA Headquarters. This research was additionally supported by NASA grants NNG05GE76G, NNX07AL75G S01, LTSA03-0000090, ATPNNG04GK55G, and ADP03-0000-092. EK acknowledges support from an Alfred P. Sloan Research Fellowship. This research has made use of NASA's Astrophysics Data System Bibliographic Services. We acknowledge use of the HEALPix, CAMB, CMBFAST, and CosmoMC packages.

\section{APPENDIX A}

\section{FISHER MATRIX ANALYSIS OF CALIBRATION AND SKY MAP FITS}

\section{A.1. Least-Squares Calibration and Sky Model Fitting}

Let $i$ be a time index in the time-ordered data. Let $g^{j}$ be parameters for the gain, $a_{l m}$ be parameters for the temperature anisotropy, and $b^{k}$ be parameters for the baseline offset.

The model of the time-ordered data (TOD) is

$$
m_{i}=g_{i}\left[\Delta T_{v i}+\Delta T_{a i}\right]+b_{i}
$$

where $i$ is a time index, $\Delta T_{v i}$ is the differential dipole signal at time step $i$, including the CMB dipole, and $\Delta T_{a i}$ is the differential anisotropy signal at time step $i$. The parameters of the model are the hourly gain and baseline values, and the sky map pixel temperatures (which goes into forming $\Delta T_{a}$. We fit for them by minimizing

$$
\chi^{2}=\sum_{i} \frac{\left(c_{i}-m_{i}\right)^{2}}{\sigma_{i}^{2}},
$$

where $c_{i}$ is the raw data, in counts, and $\sigma_{i}$ is the rms of the $i$ th observation, in counts. The Fisher matrix requires taking the second derivative of $\chi^{2}$ with respect to all parameters being fit. In order to reduce the dimensionality of the problem to something manageable, we expand the calibration and sky signal in terms of a small number of parameters. We can write

$$
\begin{gathered}
g_{i}=\sum_{j} g^{j} G_{j i}, \\
b_{i}=\sum_{k} b^{k} B_{k i}, \\
\Delta T_{a i}=\sum_{l m} a_{l m}\left[Y_{l m}\left(\hat{n}_{A i}\right)-Y_{l m}\left(\hat{n}_{B i}\right)\right],
\end{gathered}
$$

where $G$ and $B$ are functions of time (defined below), $a_{l m}$ are the harmonic coefficients of the map, and $\hat{n}_{A i}$ is the unit vector of the $A$-side feed at time step $i$, and likewise for $B$.

A reasonable set of basis functions for the gain and baseline allow for an annual modulation and a small number of higher harmonics. Note that this does not include power at the spin or precession period, which might be an important extension to consider. For now we consider the trial set

$$
G_{j i}=\left\{\begin{array}{ll}
1 & j=0 \\
\cos j \theta_{i} & j=1, \ldots, j_{\max } \\
\sin \left(j-j_{\max }\right) \theta_{i} & j=j_{\max }+1, \ldots, 2 j_{\max }
\end{array},\right.
$$

and

$$
B_{k i}=\left\{\begin{array}{ll}
1 & k=0 \\
\cos k \theta_{i} & k=1, \ldots, k_{\max } \\
\sin \left(k-k_{\max }\right) \theta_{i} & k=k_{\max }+1, \ldots, 2 k_{\max }
\end{array},\right.
$$

where $\theta=\tan ^{-1}\left(\hat{n}_{y} / \hat{n}_{x}\right)$. Here $\hat{n}$ is the unit vector from WMAP to the Sun, and the components are evaluated in ecliptic coordinates.

\section{A.2. Evaluation of the Fisher Matrix}

We wish to evaluate the second derivative

$$
\frac{1}{2} \frac{\partial^{2} \chi^{2}}{\partial p_{i} \partial p_{j}}
$$


where $p_{i}$ and $p_{j}$ are the parameters we are trying to fit. The needed first derivatives are

$$
\begin{aligned}
\frac{1}{2} \frac{\partial \chi^{2}}{\partial g j^{j^{\prime}}}= & -\sum_{i} \frac{\left(c_{i}-m_{i}\right) G_{j^{\prime} i}\left[\Delta T_{v i}+\Delta T_{a i}\right]}{\sigma_{i}^{2}} \\
& \frac{1}{2} \frac{\partial \chi^{2}}{\partial b^{k^{\prime}}}=-\sum_{i} \frac{\left(c_{i}-m_{i}\right) B_{k^{\prime} i}}{\sigma_{i}^{2}} \\
\frac{1}{2} \frac{\partial \chi^{2}}{\partial a_{l^{\prime} m^{\prime}}}= & -\sum_{i} \frac{\left(c_{i}-m_{i}\right) g_{i}\left[Y_{l^{\prime} m^{\prime}}\left(\hat{n}_{A i}\right)-Y_{l^{\prime} m^{\prime}}\left(\hat{n}_{B i}\right)\right]}{\sigma_{i}^{2}} .
\end{aligned}
$$

Then

$$
\frac{1}{2} \frac{\partial^{2} \chi^{2}}{\partial g^{j^{\prime}} \partial g^{j^{\prime \prime}}}=\sum_{i} \frac{G_{j^{\prime} i}\left[\Delta T_{v i}+\Delta T_{a i}\right] G_{j^{\prime \prime} i}\left[\Delta T_{v i}+\Delta T_{a i}\right]}{\sigma_{i}^{2}}
$$

$$
\begin{gathered}
\frac{1}{2} \frac{\partial^{2} \chi^{2}}{\partial g j^{j^{\prime}} \partial a_{l^{\prime} m^{\prime}}}=\sum_{i} \frac{g_{i}\left[Y_{l^{\prime} m^{\prime}}\left(\hat{n}_{A i}\right)-Y_{l^{\prime} m^{\prime}}\left(\hat{n}_{B i}\right)\right] G_{j^{\prime} i}\left[\Delta T_{v i}+\Delta T_{a i}\right]}{\sigma_{i}^{2}} \\
\quad+\mathcal{O} \sum_{i}\left(c_{i}-m_{i}\right) \\
\frac{1}{2} \frac{\partial^{2} \chi^{2}}{\partial g^{j^{\prime}} \partial b^{k^{\prime}}}=\sum_{i} \frac{B_{k^{\prime} i} G_{j^{\prime} i}\left[\Delta T_{v i}+\Delta T_{a i}\right]}{\sigma_{i}^{2}}
\end{gathered}
$$

$$
\begin{aligned}
& \frac{1}{2} \frac{\partial^{2} \chi^{2}}{\partial a_{l^{\prime} m^{\prime}} \partial a_{l^{\prime \prime} m^{\prime \prime}}} \\
& =\sum_{i} \frac{g_{i}\left[Y_{l^{\prime} m^{\prime}}\left(\hat{n}_{A i}\right)-Y_{l^{\prime} m^{\prime}}\left(\hat{n}_{B i}\right)\right] g_{i}\left[Y_{l^{\prime \prime} m^{\prime \prime}}\left(\hat{n}_{A i}\right)-Y_{l^{\prime \prime} m^{\prime \prime}}\left(\hat{n}_{B i}\right)\right]}{\sigma_{i}^{2}}
\end{aligned}
$$

$$
\begin{aligned}
\frac{1}{2} \frac{\partial^{2} \chi^{2}}{\partial a_{l^{\prime} m^{\prime}} \partial b^{k^{\prime}}}= & \sum_{i} \frac{g_{i} B_{k^{\prime} i}\left[Y_{l^{\prime} m^{\prime}}\left(\hat{n}_{A i}\right)-Y_{l^{\prime} m^{\prime}}\left(\hat{n}_{B i}\right)\right]}{\sigma_{i}^{2}} \\
& \frac{1}{2} \frac{\partial^{2} \chi^{2}}{\partial b^{k^{\prime}} \partial b^{k^{\prime \prime}}}=\sum_{i} \frac{B_{k^{\prime \prime} i} B_{k^{\prime} i}}{\sigma_{i}^{2}} .
\end{aligned}
$$

From this we can form the inverse covariance matrix

$$
C^{-1}=\left(\begin{array}{ccc}
\frac{1}{2} \frac{\partial^{2} \chi^{2}}{\partial g^{j^{\prime}} \partial g^{j^{\prime \prime}}} & \frac{1}{2} \frac{\partial^{2} \chi^{2}}{\partial g^{j^{\prime}} \partial a_{l^{\prime \prime} m^{\prime \prime}}} & \frac{1}{2} \frac{\partial^{2} \chi^{2}}{\partial g^{j^{\prime}} \partial b^{k^{\prime \prime}}} \\
\frac{1}{2} \frac{\partial^{2} \chi^{2}}{\partial a_{l^{\prime} m^{\prime}} \partial g^{j^{\prime \prime}}} & \frac{1}{2} \frac{\partial^{2} \chi^{2}}{\partial a_{l^{\prime} m^{\prime}} \partial a_{l^{\prime \prime} m^{\prime \prime}}} & \frac{1}{2} \frac{\partial^{2} \chi^{2}}{\partial a_{l^{\prime} m^{\prime}} \partial b^{k^{\prime \prime}}} \\
\frac{1}{2} \frac{\partial^{2} \chi^{2}}{\partial b^{k^{\prime}} \partial g^{j^{\prime \prime}}} & \frac{1}{2} \frac{\partial^{2} \chi^{2}}{\partial b^{k^{\prime}} \partial a_{l^{\prime \prime} m^{\prime \prime}}} & \frac{1}{2} \frac{\partial^{2} \chi^{2}}{\partial b^{k^{\prime}} \partial b^{k^{\prime \prime}}}
\end{array}\right),
$$

where the gain and baseline blocks are $\left(2 j_{\max }+1\right) \times\left(2 j_{\max }+1\right)$, and the sky map block is $\left(l_{\max }+1\right)^{2} \times\left(l_{\max }+1\right)^{2}$.

If we decompose $C^{-1}$ using SVD the parameter covariance matrix can be inverted to have the form

$$
C=\sum_{i} \frac{1}{w_{i}} V_{(i)} \otimes V_{(i)}
$$

where the $w_{i}$ are the singular values, and the $V_{(i)}$ are the columns of the orthogonal matrix $V$. In this form, the uncertainty in the linear combination of parameters defined by $V_{(i)}$ is $1 / w_{i}$.

\section{APPENDIX B}

\section{CALIBRATION MODEL FITTING WITH GAIN ERROR TEMPLATES}

\section{B.1. Gain Error From Calibration Dipole Error}

Consider a simple model where the input sky consists of only a pure fixed $(\mathrm{CMB})$ dipole, described by the vector $\mathbf{d}_{c}$, and a dipole modulated by the motion of WMAP with respect to the Sun, described by the time-dependent vector $\mathbf{d}_{v}(t)$. The raw data produced by an experiment observing this signal is

$$
c\left(t_{i}\right)=g\left(t_{i}\right)\left[\Delta t_{c}\left(t_{i}\right)+\Delta t_{v}\left(t_{i}\right)\right],
$$

where $c\left(t_{i}\right)$ is the TOD signal in counts, $g\left(t_{i}\right)$ is the true gain of the instrument and $\Delta t_{m}\left(t_{i}\right)$ is the differential signal produced by each dipole component $(m=c, v)$ at time $t_{i}$ given the instrument pointing at that time. Note that we have suppressed the explicit baseline and noise terms here for simplicity.

Now suppose we calibrate the instrument using an erroneous CMB dipole, $\mathbf{d}_{c}^{\prime}=r \mathbf{d}_{c}=(1+\Delta r) \mathbf{d}_{c}$, where $r$ is a number of order one (and $\Delta r \ll 1$ so we can ignore terms of order $\Delta r^{2}$ ). The fit gain, $g_{f}(t)$, will then roughly have the form

$$
g_{f}(t)=\frac{c(t)}{\left|\mathbf{d}_{c}^{\prime}+\mathbf{d}_{v}(t)\right|}=g(t) \frac{\left|\mathbf{d}_{c}+\mathbf{d}_{v}(t)\right|}{\left|r \mathbf{d}_{c}+\mathbf{d}_{v}(t)\right|},
$$

where the vertical bars indicate vector magnitude. Now define $\mathbf{d} \equiv \mathbf{d}_{c}+\mathbf{d}_{v}$ and expand to first order in $\Delta r$ to obtain

$$
g_{f}(t)=g(t)\left[1-\Delta r \frac{\mathbf{d}(t) \cdot \mathbf{d}_{c}}{\mathbf{d}(t) \cdot \mathbf{d}(t)}\right] .
$$

Note that the term $\left(\mathbf{d} \cdot \mathbf{d}_{c}\right) /(\mathbf{d} \cdot \mathbf{d})$ is dominated by a constant component of order $d_{c}^{2} /\left(d_{c}^{2}+d_{v}^{2}\right) \sim 0.99$, followed by an annually modulated term that is suppressed by a factor of order $d_{v} / d_{c}$. Thus an erroneous calibration dipole induces a specific error in the fit gain that can be identified and corrected for, assuming the time dependence of the true gain is orthogonal to this form.

\section{B.2. Gain Model Fitting}

In theory, the way to do this is as follows. We have a set of data in the form of the fit gains, $g_{f, i}$ for each calibration sequence $i$, and we have a gain model, $G\left(t ; p_{n}\right)$, which is a function of time and a set of model parameters $p_{n}$. Ideally we would like to fit the model to the true gain, $g(t)$, but since we do not know the true gain, the next best thing is to modify the gain model to have the same modulation form as the dipole gains have and to fit for this modulation simultaneously with the other gain model parameters. Thus $\chi^{2}$ takes the form

$$
\chi^{2}=\sum_{i} \frac{\left[g_{i}-G_{i}\left(p_{n}\right)\right]^{2}}{\sigma_{i}^{2}}=\sum_{i} \frac{\left[g_{f, i}-G_{i}\left(p_{n}\right)\left(1-\Delta r f_{d, i}\right)\right]^{2}}{\sigma_{i}^{2}},
$$

where $f_{d, i} \equiv(\mathbf{d} \cdot \mathbf{d} c) /(\mathbf{d} \cdot \mathbf{d})$ evaluated at time $t_{i}$, or is a function generated from simulations.

Since the system is nonlinear, it must be minimized using a suitable nonlinear least squares routine. However, we can analyze the parameter covariance matrix directly by explicitly evaluating the second derivative of $\chi^{2}$ with respect to the model parameters

$$
C^{-1}=\frac{1}{2} \frac{\partial^{2} \chi^{2}}{\partial p_{j} \partial p_{k}}
$$


First, the necessary first derivatives are compiled:

$$
\frac{1}{2} \frac{\partial \chi^{2}}{\partial \Delta r}=\sum_{i} \frac{\left[g_{f, i}-G_{i}\left(p_{n}\right)\left(1-\Delta r f_{d, i}\right)\right]\left(G_{i} f_{d, i}\right)}{\sigma_{i}^{2}}
$$

$$
\begin{aligned}
& \frac{1}{2} \frac{\partial \chi^{2}}{\partial p_{m}}= \\
& \sum_{i} \frac{\left[g_{f, i}-G_{i}\left(p_{n}\right)\left(1-\Delta r f_{d, i}\right)\right]\left(-\partial G_{i} / \partial p_{m}\right)\left(1-\Delta r f_{d, i}\right)}{\sigma_{i}^{2}}
\end{aligned}
$$

(we evaluate the individual $\partial G / \partial p_{m}$ terms below). Next the various second derivatives are

$$
\frac{1}{2} \frac{\partial^{2} \chi^{2}}{\partial \Delta r \partial \Delta r}=\sum_{i} \frac{\left(G_{i} f_{d, i}\right)\left(G_{i} f_{d, i}\right)}{\sigma_{i}^{2}}
$$

$$
\begin{aligned}
& \frac{1}{2} \frac{\partial^{2} \chi^{2}}{\partial \Delta r \partial p_{m}}= \\
& \sum_{i} \frac{\left(G_{i} f_{d, i}\right)\left(-\partial G_{i} / \partial p_{m}\right)\left(1-\Delta r f_{d, i}\right)}{\sigma_{i}^{2}}+\mathcal{O} \sum_{i}\left(g_{i}-G_{i}\right)
\end{aligned}
$$

$$
\begin{aligned}
\frac{1}{2} \frac{\partial^{2} \chi^{2}}{\partial p_{m} \partial p_{n}}= & \sum_{i} \frac{\left(\partial G_{i} / \partial p_{m}\right)\left(1-\Delta r f_{d, i}\right)\left(\partial G_{i} / \partial p_{n}\right)\left(1-\Delta r f_{d, i}\right)}{\sigma_{i}^{2}} \\
& +\mathcal{O} \sum_{i}\left(g_{i}-G_{i}\right) .
\end{aligned}
$$

In the last two expressions, we neglect the term proportional to $\partial^{2} G / \partial p_{m} \partial p_{n}$ because the prefactor of $\left(g_{i}-G_{i}\right)$ is statistically zero for the least squares solution.

Finally, we evaluate the $\partial G / \partial p_{m}$ terms. The gain model has the form (Jarosik et al. 2007)

$$
G_{i}=\alpha \frac{\bar{V}\left(t_{i}\right)-V_{0}-\beta\left(T_{\mathrm{RXB}}\left(t_{i}\right)-T_{\mathrm{RXB}}^{0}\right)}{T_{\mathrm{FPA}}\left(t_{i}\right)-T_{\mathrm{FPA}}^{0}},
$$

where $T_{\mathrm{RXB}}^{0} \equiv 290 \mathrm{~K}$, and $\alpha, V_{0}$, and $T_{\mathrm{FPA}}^{0}$ are parameters to be fit. The necessary first derivatives are

$$
\begin{gathered}
\partial G_{i} / \partial \alpha=\frac{\bar{V}\left(t_{i}\right)-V_{0}-\beta\left(T_{\mathrm{RXB}}\left(t_{i}\right)-T_{\mathrm{RXB}}^{0}\right)}{T_{\mathrm{FPA}}\left(t_{i}\right)-T_{\mathrm{FPA}}^{0}}, \\
\partial G_{i} / \partial V_{0}=\frac{-\alpha}{T_{\mathrm{FPA}}\left(t_{i}\right)-T_{\mathrm{FPA}}^{0}}, \\
\partial G_{i} / \partial \beta=\frac{-\alpha\left(T_{\mathrm{RXB}}\left(t_{i}\right)-T_{\mathrm{RXB}}^{0}\right)}{T_{\mathrm{FPA}}\left(t_{i}\right)-T_{\mathrm{FPA}}^{0}},
\end{gathered}
$$

$$
\partial G_{i} / \partial T_{\mathrm{FPA}}^{0}=\alpha \frac{\bar{V}\left(t_{i}\right)-V_{0}-\beta\left(T_{\mathrm{RXB}}\left(t_{i}\right)-T_{\mathrm{RXB}}^{0}\right)}{\left(T_{\mathrm{FPA}}\left(t_{i}\right)-T_{\mathrm{FPA}}^{0}\right)^{2}} .
$$

\section{REFERENCES}

Astier, P., et al. 2006, A\&A, 447, 31

Barnes, C., et al. 2003, ApJS, 148, 51

Bennett, C. L., et al. 2003, ApJ, 583, 1

Benoît, A., et al. 2003, A\&A, 399, L25

Born, M., \& Wolf, E. 1980, Principles of Optics (6th ed.; Oxford: Pergamon) Cole, S., et al. 2005, MNRAS, 362, 505

Copi, C. J., Huterer, D., Schwarz, D. J., \& Starkman, G. D. 2007, Phys. Rev. D, 75,023507

Creminelli, P., Nicolis, A., Senatore, L., Tegmark, M., \& Zaldarriaga, M. 2006, J. Cosmol. Astropart. Phys., JCAP05 (2006) 004

Dunkley, J., et al. 2009, ApJS, 180, 306

Eisenstein, D. J., et al. 2005, ApJ, 633, 560

Eriksen, H. K., Banday, A. J., Górski, K. M., Hansen, F. K., \& Lilje, P. B. 2007a, ApJ, 660, L81

Eriksen, H. K., Jewell, J. B., Dickinson, C., Banday, A. J., Gorski, K. M., \& Lawrence, C. R. 2008, ApJ, 676, 10

Eriksen, H. K., et al. 2007b, ApJ, 656, 641

Freedman, W. L., et al. 2001, ApJ, 553, 47

Gold, B., et al. 2009, ApJS, 180, 265

Gorski, K. M., Hivon, E., Banday, A. J., Wandelt, B. D., Hansen, F. K., Reinecke, M., \& Bartlemann, M. 2005, ApJ, 622, 759

Halverson, N. W., et al. 2002, ApJ, 568, 38

Hill, R., et al. 2009, ApJS, 180, 246

Hinshaw, G., et al. 2003, ApJS, 148, 63

Hinshaw, G., et al. 2007, ApJS, 170, 288

Hunt, P., \& Sarkar, S. 2007, Phys. Rev. D, 76, 123504

Jarosik, N., et al. 2003, ApJS, 145, 413

Jarosik, N., et al. 2007, ApJS, 170, 263

Kogut, A., et al. 1996, ApJ, 470, 653

Komatsu, E., et al. 2009, ApJS, 180, 330

Land, K., \& Magueijo, J. 2007, MNRAS, 378, 153

Lee, A. T., et al. 2001, ApJ, 561, L1

Limon, M., et al. 2008, Wilkinson Microwave Anisotropy Probe (WMAP): Explanatory Supplement, http://lambda.gsfc.nasa.gov/data/map/doc/MAP supplement.pdf

Mather, J. C., Fixsen, D. J., Shafer, R. A., Mosier, C., \& Wilkinson, D. T. 1999, ApJ, 512, 511

Miller, A. D., et al. 1999, ApJ, 524, L1

Netterfield, C. B., et al. 2002, ApJ, 571, 604

Nolta, M. R., et al. 2009, ApJS, 180, 296

Page, L., et al. 2007, ApJS, 170, 335

Pearson, T. J., et al. 2003, ApJ, 591, 556

Peiris, H. V., et al. 2003, ApJS, 148, 213

Percival, W. J., Cole, S., Eisenstein, D. J., Nichol, R. C., Peacock, J. A., Pope, A. C., \& Szalay, A. S. 2007, MNRAS, 381, 1053

Percival, W. J., et al. 2001, MNRAS, 327, 1297

Riess, A. G., et al. 2007, ApJ, 659, 98

Scott, P. F., et al. 2003, MNRAS, 341, 1076

Shafieloo, A., \& Souradeep, T. 2008, Phys. Rev. D, 78, 023511

Spergel, D. N., et al. 2003, ApJS, 148, 175

Spergel, D. N., et al. 2007, ApJS, 170, 377

Tegmark, M., et al. 2004, Phys. Rev. D, 69, 103501

Tegmark, M., et al. 2006, Phys. Rev. D, 74, 123507

Wood-Vasey, W. M., et al. 2007, ApJ, 666, 694

Wright, E. L. 2007, arXiv:astro-ph/0703640

Wright, E. L., et al. 2009, ApJS, 180, 283

Yadav, A. P. S., Komatsu, E., Wandelt, B. D., Liguori, M., Hansen, F. K., \& Matarrese, S. 2007, arXiv:0711.4933

Yadav, A. P. S., \& Wandelt, B. D. 2008, Phys. Rev. Lett., 100, 181301 